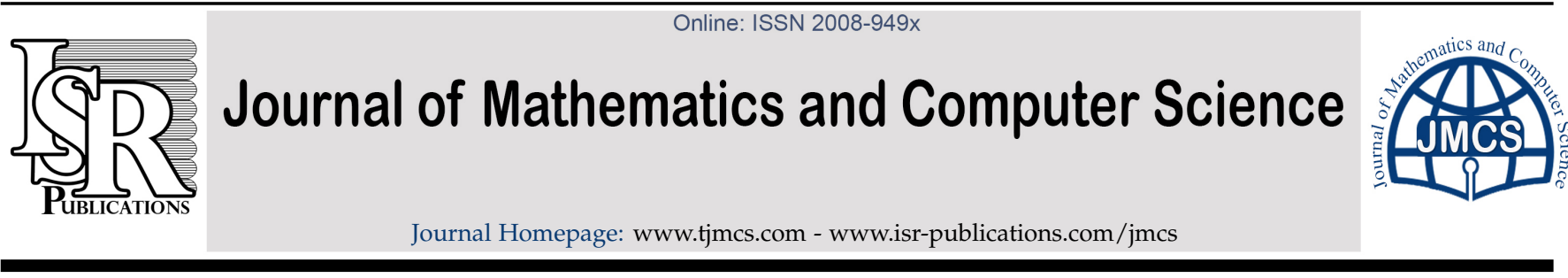

\title{
Bifurcations of heteroclinic loops with nonresonant eigenvalues
}

\author{
Zheng Guo ${ }^{\mathrm{a}}$, Yinlai Jin ${ }^{\mathrm{a}, *}$, Yuerang Gao ${ }^{\mathrm{a}, \mathrm{b}}$, Dandan Xie $\mathrm{X}^{\mathrm{a}, \mathrm{c}}$ \\ ${ }^{a}$ School of Science, Linyi University, Linyi, Shandong, 276005, China. \\ ${ }^{b}$ Lanling County First Middle School, Lanling, Shangdong, 277700, China. \\ 'School of Mathematical Sciences, Shandong Normal University, Jinan, 250014, China.
}

\begin{abstract}
In this paper, we use the way of local coordinates instead of the Floquet method to study the problems of homoclinic and periodic orbits bifurcated from heteroclinic loop for high-dimensional system. Under some transversal conditions and the non-twisted or twisted conditions, we discuss the existence, uniqueness, coexistence, and non-coexistence of 1-periodic orbit, 1-homoclinic orbit, and 1-heteroclinic orbit near the heteroclinic loop. We get some general conclusions only under the basic hypotheses, and the other conclusions under the two hyperbolic ratios of the heteroclinic loop are greater than 1 . Meanwhile, the bifurcation surfaces and existence regions are given. (C)2017 all rights reserved.
\end{abstract}

Keywords: Heteroclinic loop, Poincaré map, periodic orbit, bifurcation surface. 2010 MSC: 34C23, 34C37, 37C29.

\section{Introduction and hypotheses}

In recent years, the bifurcation problems of heteroclinic orbits in high dimensional space were studied and many results were obtained (see [1-3, 6-9]). In [10], Zhu and Xia studied the bifurcation problems of heteroclinic loops with two hyperbolic saddle points by generalizing the Floquet method and exponential dichotomy. In [5], Jin et al. studied the bifurcations of non-twisted heteroclinic loop with resonant eigenvalues. In [4], Jin and Zhu studied the bifurcations of rough heteroclinic loop with two saddle points for the hyperbolic ratios $\beta_{i}, i=1,2$, satisfying $\beta_{1}>1, \beta_{2}<1$ and $\beta_{1} \beta_{2}<1$.

In this paper, we use the way of local coordinates instead of the Floquet method to study the problems of homoclinic and periodic orbits bifurcated from heteroclinic loop for high dimensional system. Under some transversal conditions and the non-twisted or twisted conditions, we discuss the existence, uniqueness, coexistence and non-coexistence of the 1-periodic orbit, 1-homoclinic orbit, and 1-heteroclinic orbit near the heteroclinic loop. We obtain some general conclusions only under the basic assumptions, and the other conclusions under hyperbolic ratio $\beta_{i}$ satisfying $\beta_{i}>1, i=1,2$. Moreover, we give the bifurcation surfaces and their relative positions and the existence regions of 1-periodic orbit.

\footnotetext{
${ }^{*}$ Corresponding author

Email addresses: guozheng@lyu.edu.cn (Zheng Guo), jinyinlai@lyu.edu.cn (Yinlai Jin), yueranggao@sina.com (Yuerang Gao), xiedandan01@sina.com (Dandan Xie)
}

doi:10.22436/jmcs.017.01.11 
Consider the following $\mathrm{C}^{\mathrm{r}}$ system

$$
\dot{z}=f(z)+g(z, \mu)
$$

and its unperturbed system

$$
\dot{z}=f(z),
$$

where $r \geqslant 5, z \in R^{m+n}, \mu \in R^{l}, l \geqslant 3,0 \leqslant|\mu| \ll 1$, and $g(z, 0)=0$.

(H1) $z=p_{i}, i=1,2$ are hyperbolic critical points of system (1.2), $f\left(p_{i}\right)=0, g\left(p_{i}, \mu\right)=0$, the stable manifold $W_{i}^{s}$ and the unstable manifold $W_{i}^{u}$ of $z=p_{i}$ are m-dimensional and $n$-dimensional, respectively. Moreover, $-\rho_{i}^{1}$ and $\lambda_{i}^{1}$ are the simple real eigenvalues of $D_{z} f\left(p_{i}\right)$ such that any other eigenvalue $\sigma$ of $D_{z} f\left(p_{i}\right)$ satisfies either $\operatorname{Re} \sigma<-\rho_{i}^{0}<-\rho_{i}^{1}<0$ or $0<\lambda_{i}^{1}<\lambda_{i}^{0}<\operatorname{Re} \sigma$, where $\rho_{i}^{0}$ and $\lambda_{i}^{0}$ are some positive constants.

(H2) System (1.2) has a heteroclinic loop $\Gamma=\Gamma_{1} \cup \Gamma_{2}$, where $\Gamma_{\mathfrak{i}}=\left\{z=\gamma_{\mathfrak{i}}(\mathrm{t}): \mathrm{t} \in \mathrm{R}\right\}, \gamma_{\mathfrak{i}}(+\infty)=$ $\gamma_{i+1}(-\infty)=p_{i+1}, \gamma_{3}(t)=\gamma_{1}(t), p_{3}=p_{1}$. For any point $P_{i} \in \Gamma_{i}, \operatorname{dim}\left(T_{P_{i}} W_{i}^{u} \cap T_{P_{i}} W_{i+1}^{s}\right)=1$, where $W_{3}^{s}=W_{1}^{s}, T_{P_{i}} W_{i}^{u}$ is the tangent space of $W_{i}^{u}$ at $P_{i}$, and $T_{P_{i}} W_{i+1}^{s}$ is the tangent space of $W_{i+1}^{s}$ at $P_{i}$.

(H3) Define $e_{i}^{ \pm}=\lim _{t \rightarrow \mp \infty} \frac{\dot{\gamma}_{i}(t)}{\left|\dot{\gamma}_{i}(t)\right|}$, then $e_{i}^{+} \in \mathrm{T}_{p_{i}} W_{i}^{u}$ and $e_{i}^{-} \in \mathrm{T}_{p_{i+1}} W_{i+1}^{s}$ are unit eigenvectors corresponding to $\lambda_{i}^{1}$ and $-\rho_{i+1}^{1}$, respectively. Denote $\operatorname{span}\left(T_{p_{i}} W_{i}^{u u}, e_{i}^{+}\right)=T_{p_{i}} W_{i}^{u}, \operatorname{span}\left(T_{p_{i}} W_{i}^{s s}, e_{i+1}^{-}\right)=$ $T_{p_{i}} W_{i}^{s}$, where $W_{i}^{\text {uu }}$ and $W_{i}^{\text {ss }}$ are the strong unstable manifolds and the strong stable manifolds of $p_{i}$, respectively, $T_{p_{i}} W_{i}^{\text {uu }}$ is the tangent space of $W_{i}^{\text {uu }}$ at $p_{i}$, and $T_{p_{i}} W_{i}^{s s}$ is the tangent space of $W_{i}^{s s}$ at $p_{i}$. That is, $T_{p_{i}} W_{i}^{u u}$ is the generalized eigenspace corresponding to all the eigenvalues with larger real part than $\lambda_{i}^{0}, T_{p_{i}} W_{i}^{s s}$ is the generalized eigenspace corresponding to all the eigenvalues with smaller real part than $-\rho_{i}^{0}$. The following strong inclination hold:

$$
\begin{aligned}
& \lim _{t \rightarrow+\infty}\left(T_{\gamma_{i}(t)} W_{i}^{u}+T_{\gamma_{i}(t)} W_{i+1}^{s}\right)=T_{p_{i+1}} W_{i+1}^{u u} \oplus T_{p_{i+1}} W_{i+1}^{s}, \\
& \lim _{t \rightarrow-\infty}\left(T_{\gamma_{i}(t)} W_{i}^{u}+T_{\gamma_{i}(t)} W_{i+1}^{s}\right)=T_{p_{i}} W_{i}^{u} \oplus T_{p_{i}} W_{i}^{s s} .
\end{aligned}
$$

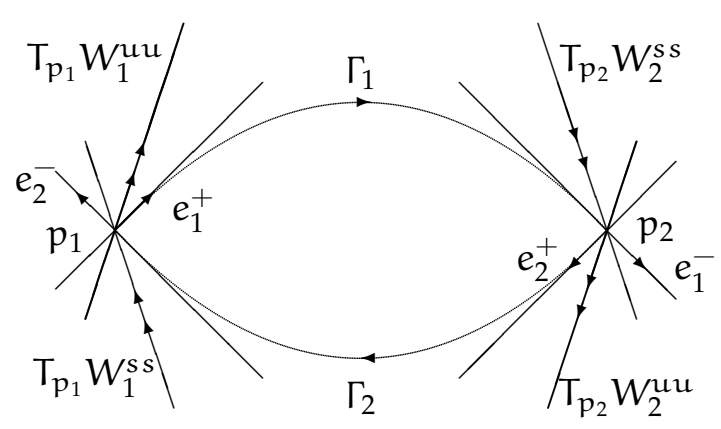

Figure 1

\section{Local Coordinates}

In this section, we will establish a suitable system of local coordinates in the neighborhood of heteroclinic loop $\Gamma$. This method is similar to that of in [4]. Based on the analysis of the Poincaré maps defined on some local transversal sections of $\Gamma$, we need to normalize (1.1) in some small enough neighborhood $U_{i}$ of $p_{i}$, and set up a system of local coordinates near the loop $\Gamma$.

Suppose that $(\mathrm{H} 1)-(\mathrm{H} 3)$ hold, then, it is well-known that there always exists a $\mathrm{C}^{\mathrm{r}}$ transformation such that system (1.1) has the following form in $\mathrm{U}_{i}$ : 


$$
\left\{\begin{array}{l}
\dot{x}=\left[\lambda_{i}^{1}(\mu)+\text { h.o.t. }\right] x+\mathrm{O}(\mathrm{u})[\mathrm{O}(\mathrm{y})+\mathrm{O}(v)], \\
\dot{y}=\left[-\rho_{i}^{1}(\mu)+\text { h.o.t. }\right] y+\mathrm{O}(v)[\mathrm{O}(\mathrm{x})+\mathrm{O}(\mathrm{u})], \\
\dot{u}=\left[\mathrm{B}_{i}^{1}(\mu)+\text { h.o.t. }\right] \mathrm{u}+\mathrm{O}(\mathrm{x})[\mathrm{O}(\mathrm{x})+\mathrm{O}(\mathrm{y})+\mathrm{O}(v)], \\
\dot{v}=\left[-\mathrm{B}_{i}^{2}(\mu)+\text { h.o.t. }\right] v+\mathrm{O}(\mathrm{y})[\mathrm{O}(\mathrm{x})+\mathrm{O}(\mathrm{y})+\mathrm{O}(\mathrm{u})],
\end{array}\right.
$$

for $|\mu|$ small enough, where $\lambda_{i}^{1}(0)=\lambda_{i}^{1}, \rho_{i}^{1}(0)=\rho_{i}^{1}, \operatorname{Re} \sigma\left(B_{i}^{1}(0)\right)>\lambda_{i}^{0}, \operatorname{Re} \sigma\left(-B_{i}^{2}(0)\right)<-\rho_{i}^{0}, z=\left(x, y, u^{*}, v^{*}\right)^{*}$, $x \in R^{1}, y \in R^{1}, u \in R^{n-1}, v \in R^{m-1}, i=1,2$. The sign $*$ means transposition, the "h.o.t." means higher order term and the system (2.1) is $\mathrm{C}^{\mathrm{r}-1}$.

In other words, we have assumed that the local unstable, stable, strong unstable and strong stable manifolds of $p_{i}$ in $U_{i}$ are given by

$$
\begin{array}{rlrl}
W_{i}^{u} & =\{z: y=0, v=0\}, & W_{i}^{s} & =\{z: x=0, u=0\}, \\
W_{i}^{u u} & =\{z: x=0, y=0, v=0\}, & W_{i}^{s s} & =\{z: x=0, y=0, u=0\}, \\
\Gamma \cap W_{i}^{u} & =\{z: y=0, u=u(x), v=0\}, & \Gamma \cap W_{i}^{s}=\{z: x=0, u=0, v=v(y)\},
\end{array}
$$

where $\dot{u}(0)=u(0)=0, \dot{v}(0)=v(0)=0$.

Taking moments $T_{i}^{0}$ and $T_{i}^{1}$ such that $\gamma_{i}\left(-T_{i}^{0}\right)=\left(\delta, 0, \delta_{\mathfrak{u}_{i},}^{*} 0^{*}\right)^{*}, \gamma_{i}\left(T_{i}^{1}\right)=\left(0, \delta, 0^{*}, \delta_{v_{i}}^{*}\right)^{*}$, where $T_{i}^{0}$ and $\mathrm{T}_{i}^{1}$ are large enough and $\delta$ is small enough such that $\left\{\left(x, y, u^{*}, v^{*}\right)^{*}:|x|,|y|,|u|,|v|<2 \delta\right\} \in U_{i}$. Obviously, $\left|\delta_{u_{i}}\right|=o(\delta),\left|\delta_{v_{i}}\right|=o(\delta)$.

Consider the linear variational system

$$
\dot{z}=\operatorname{Df}\left(\mathrm{r}_{\mathfrak{i}}(\mathrm{t})\right) z
$$

and its adjoint system

$$
\dot{\varphi}=-\left(\mathrm{Df}\left(\mathrm{r}_{\mathrm{i}}(\mathrm{t})\right)\right)^{*} \varphi .
$$

Under the hypothesis (H1)-(H3), both (2.2) and (2.3) have exponential dichotomies in $\mathrm{R}^{+}$and $\mathrm{R}^{-}$. (See $[9,10])$

According to $[4,5],(2.2)$ has a fundamental solution matrix $Z_{\mathfrak{i}}(t)=\left(z_{\mathfrak{i}}^{1}(t), z_{\mathfrak{i}}^{2}(t), z_{\mathfrak{i}}^{3}(t), z_{\mathfrak{i}}^{4}(t)\right)$, such that

$$
\begin{aligned}
& z_{\mathfrak{i}}^{1}(\mathrm{t}) \in\left(\mathrm{T}_{\gamma_{\mathfrak{i}}(\mathrm{t})} \mathrm{W}_{\mathfrak{i}}^{\mathrm{u}}\right)^{\mathrm{c}} \cap\left(\mathrm{T}_{\gamma_{\mathfrak{i}}(\mathrm{t})} \mathrm{W}_{\mathrm{i}+1}^{\mathrm{s}}\right)^{\mathrm{c}}, \\
& z_{i}^{2}(\mathrm{t})=-\frac{\dot{\gamma}_{i}(\mathrm{t})}{\left|\dot{\gamma}_{i}^{\mathrm{y}}\left(\mathrm{T}_{i}^{1}\right)\right|} \in \mathrm{T}_{\gamma_{\mathfrak{i}}(\mathrm{t})} \mathrm{W}_{i}^{\mathrm{u}} \cap \mathrm{T}_{\gamma_{\mathfrak{i}}(\mathrm{t})} \mathrm{W}_{i+1}^{\mathrm{s}} \text {, } \\
& z_{i}^{3}(t)=\left(z_{i}^{3,1}(t), \ldots, z_{i}^{3, n-1}(t)\right) \in T_{\gamma_{i}(t)} W_{i}^{u} \cap\left(T_{\gamma_{i}(t)} W_{i+1}^{s}\right)^{c}=T_{\gamma_{i}(t)} W_{i}^{u u}, \\
& z_{i}^{4}(t)=\left(z_{i}^{4,1}(t), \ldots, z_{i}^{4, m-1}(t)\right) \in\left(T_{\gamma_{i}(t)} W_{i}^{u}\right)^{c} \cap T_{\gamma_{i}(t)} W_{i+1}^{s}=T_{\gamma_{i}(t)} W_{i+1}^{s s} \text {, } \\
& Z_{i}\left(-T_{i}^{0}\right)=\left(\begin{array}{cccc}
w_{i}^{11} & w_{i}^{21} & 0 & w_{i}^{41} \\
w_{i}^{12} & 0 & 0 & w_{i}^{42} \\
w_{i}^{13} & w_{i}^{23} & I & w_{i}^{43} \\
0 & 0 & 0 & w_{i}^{44}
\end{array}\right), Z_{i}\left(T_{i}^{1}\right)=\left(\begin{array}{cccc}
1 & 0 & w_{i}^{31} & 0 \\
0 & 1 & w_{i}^{32} & 0 \\
0 & 0 & w_{i}^{33} & 0 \\
w_{i}^{14} & w_{i}^{24} & w_{i}^{34} & I
\end{array}\right) \text {, }
\end{aligned}
$$

where $W_{3}^{s s}=W_{1}^{s s}, w_{i}^{21}<0, w_{i}^{12} \neq 0,\left\|w_{i}^{44}\right\| \neq 0,\left\|w_{i}^{33}\right\| \neq 0$. Moreover, for $\delta$ small enough, $\left\|w_{i}^{1 j}\left(w_{i}^{12}\right)^{-1}\right\| \ll$ $1, j \neq 2 ;\left\|w_{i}^{2 j}\left(w_{i}^{21}\right)^{-1}\right\| \ll 1, j=3,4 ;\left\|w_{i}^{3 j}\left(w_{i}^{33}\right)^{-1}\right\| \ll 1, j \neq 3 ;\left\|w_{i}^{4 j}\left(w_{i}^{44}\right)^{-1}\right\| \ll 1, j \neq 4$.

Denoting $\triangle_{i}=\frac{w_{i}^{12}}{\left|w_{i}^{12}\right|}$, we say that $\Gamma_{i}$ is non-twisted (twisted) when $\triangle_{i}=1\left(\triangle_{i}=-1\right)$.

Thus, we may regard $z_{i}^{1}(t), z_{i}^{2}(t), z_{i}^{3}(t), z_{i}^{4}(t)$ as a local coordinate system along $\Gamma_{i}$.

It was clear that $\Phi_{i}(t)=\left(\varphi_{i}^{1}(t), \varphi_{i}^{2}(t), \varphi_{i}^{3}(t), \varphi_{i}^{4}(t)\right)=\left(Z_{i}^{-1}(t)\right)^{*}$ is a fundamental solution matrix of (2.3), and $\varphi_{i}^{1}(t)$ is bounded and tends to zero exponentially as $t \rightarrow \pm \infty$ (see $[4,9,10]$ ).

\section{Poincaré maps and bifurcation equations}

Now we set up the Poincaré maps. First, we use $\left(z_{i}^{1}(t), z_{i}^{2}(t), z_{i}^{3}(t), z_{i}^{4}(t)\right)$ to define the Poincaré sections. Let $N_{i}=\left(n_{i}^{1}, 0,\left(n_{i}^{3}\right)^{*},\left(n_{i}^{4}\right)^{*}\right)^{*}, n_{i}^{3}=\left(n_{i}^{3,1}, \ldots, n_{i}^{3, n-1}\right)^{*}, n_{i}^{4}=\left(n_{i}^{4,1}, \ldots, n_{i}^{4, m-1}\right)^{*}$, and $h_{i}(t)=\gamma_{i}(t)+$ 
$Z_{i}(t) N_{i}$ in the neighborhood of $\Gamma$. Thus we define

$$
S_{i}^{0}=\left\{z=h_{\mathfrak{i}}\left(-T_{i}^{0}\right):|x|,|y|,|u|,|v|<2 \delta\right\}, S_{i}^{1}=\left\{z=h_{\mathfrak{i}}\left(T_{i}^{1}\right):|x|,|y|,|u|,|v|<2 \delta\right\}
$$

to be cross sections of $\Gamma_{i}$ at $t=-T_{i}^{0}$ and $t=T_{i}^{1}$, respectively, where $\delta$ is small enough such that $S_{i}^{0} \subset U_{i}$, $\mathrm{S}_{\mathrm{i}}^{1} \subset \mathrm{U}_{\mathrm{i}+1}, \mathrm{U}_{3}=\mathrm{U}_{1}$.

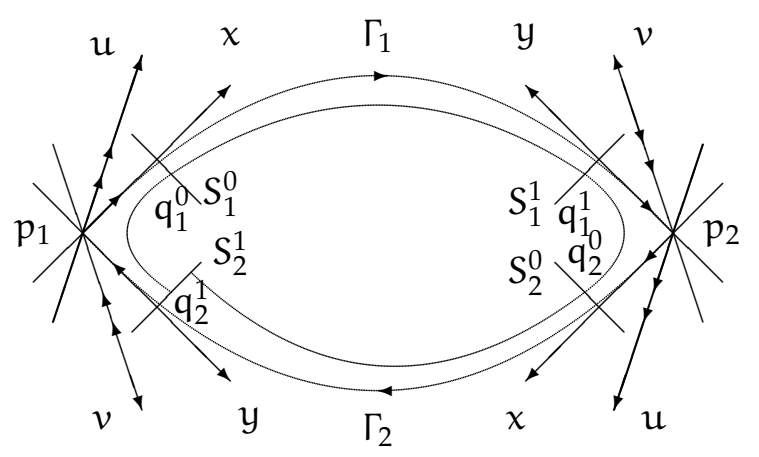

Figure 2

We consider the map $F_{i}^{1}: q_{i}^{0} \in S_{i}^{0} \rightarrow q_{i}^{1} \in S_{i}^{1}$. Let

$$
\begin{aligned}
& q_{i}^{0}=\left(x_{i}^{0}, y_{i}^{0},\left(u_{i}^{0}\right)^{*},\left(v_{i}^{0}\right)^{*}\right)^{*}=\gamma_{i}\left(-T_{i}^{0}\right)+Z_{i}\left(-T_{i}^{0}\right) N_{i}^{0}, N_{i}^{0}=\left(n_{i}^{0,1}, 0,\left(n_{i}^{0,3}\right)^{*},\left(n_{i}^{0,4}\right)^{*}\right)^{*}, \\
& q_{i}^{1}=\left(x_{i}^{1}, y_{i}^{1},\left(u_{i}^{1}\right)^{*},\left(v_{i}^{1}\right)^{*}\right)^{*}=\gamma_{i}\left(T_{i}^{1}\right)+Z_{i}\left(T_{i}^{1}\right) N_{i}^{1}, N_{i}^{1}=\left(n_{i}^{1,1}, 0,\left(n_{i}^{1,3}\right)^{*},\left(n_{i}^{1,4}\right)^{*}\right)^{*} .
\end{aligned}
$$

According to the expressions of $Z_{i}\left(-T_{i}^{0}\right), Z_{i}\left(T_{i}^{1}\right), i=1,2$, we have $x_{i}^{0} \approx \delta, y_{i}^{1} \approx \delta$, and

$$
\begin{aligned}
& \left\{\begin{array}{l}
n_{i}^{1,1}=x_{i}^{1}-w_{i}^{31}\left(w_{i}^{33}\right)^{-1} u_{i}^{1}, \\
n_{i}^{1,3}=\left(w_{i}^{33}\right)^{-1} u_{i}^{1}, \\
n_{i}^{1,4}=-w_{i}^{14} x_{i}^{1}+\left(w_{i}^{14} w_{i}^{31}+w_{i}^{24} w_{i}^{32}-w_{i}^{34}\right)\left(w_{i}^{33}\right)^{-1} u_{i}^{1}+v_{i}^{1}-\delta_{v_{i},}
\end{array}\right. \\
& \left\{\begin{array}{l}
n_{i}^{0,1}=\left(w_{i}^{12}\right)^{-1}\left(y_{i}^{0}-w_{i}^{42}\left(w_{i}^{44}\right)^{-1} v_{i}^{0}\right), \\
n_{i}^{0,3}=u_{i}^{0}-\delta_{u_{i}}-w_{i}^{13}\left(w_{i}^{12}\right)^{-1} y_{i}^{0}+\left(w_{i}^{13}\left(w_{i}^{12}\right)^{-1} w_{i}^{42}-w_{i}^{43}\right)\left(w_{i}^{44}\right)^{-1} v_{i}^{0}, \\
n_{i}^{0,4}=\left(w_{i}^{44}\right)^{-1} v_{i}^{0} .
\end{array}\right.
\end{aligned}
$$

Take a coordinate transformation $z=h_{i}(t), t \in\left[-T_{i}^{0}, T_{i}^{1}\right]$, substituting it into (1.1), and using $\dot{\gamma}_{i}(t)=$ $f\left(\gamma_{i}(t)\right), \dot{Z}_{i}(t)=\operatorname{Df}\left(\gamma_{i}(t)\right) Z_{i}(t)$, one can see that (1.1) is transformed into the following form:

$$
\dot{\mathfrak{n}}_{i}^{j}=\varphi_{i}^{j *}(t) g_{\mu}\left(\gamma_{i}(t), 0\right) \mu+\text { h.o.t., } i=1,2, j=1,3,4 \text {. }
$$

Thus, the map $F_{i}^{1}: S_{i}^{0} \rightarrow S_{i}^{1}$ is defined by $N_{i}\left(-T_{i}^{0}\right) \rightarrow N_{i}\left(T_{i}^{1}\right)$,

$$
n_{i}^{j}\left(T_{i}^{1}\right)=n_{i}^{j}\left(-T_{i}^{0}\right)+M_{i}^{j} \mu+\text { h.o.t., } i=1,2, j=1,3,4 \text {. }
$$

That is,

$$
n_{i}^{1, j}=n_{i}^{0, j}+M_{i}^{j} \mu+\text { h.o.t., } i=1,2, j=1,3,4,
$$

where $M_{i}^{j}=\int_{-\infty}^{+\infty} \varphi_{i}^{j *}(t) g_{\mu}\left(\gamma_{i}(t), 0\right) d t, i=1,2, j=1,3,4$, (see $\left.[4,9,10]\right)$.

Next, we consider the map $F_{i}^{0}: q_{i-1}^{1} \in S_{i-1}^{1} \rightarrow q_{i}^{0} \in S_{i}^{0}$, where $q_{i-1}^{1}=\left(x_{i-1}^{1}, y_{i-1}^{1},\left(u_{i-1}^{1}\right)^{*},\left(v_{i-1}^{1}\right)^{*}\right)^{*}$, $q_{i}^{0}=\left(x_{i}^{0}, y_{i}^{0},\left(u_{i}^{0}\right)^{*},\left(v_{i}^{0}\right)^{*}\right)^{*}$. 
Let $\eta_{i}(\mu)=\min \left\{\rho_{i}^{1}(\mu), \lambda_{i}^{1}(\mu)\right\}, \eta_{i}=\eta_{i}(0), \rho_{i}^{1}=\rho_{i}^{1}(0), \lambda_{i}^{1}=\lambda_{i}^{1}(0)$ and $\tau_{i}$ be the flying time from $q_{i-1}^{1}$ to $q_{i}^{0}, s_{i}=e^{-\eta_{i}(\mu) \tau_{i}}$. Neglecting all higher order terms we get $($ see $[4,5])$

$$
\begin{array}{ll}
x_{i-1}^{1} \approx s_{i}^{\frac{\lambda_{i}^{1}(\mu)}{\eta_{i}(\mu)}} x_{i}^{0} \approx s_{i}^{\frac{\lambda_{i}^{1}(\mu)}{\eta_{i}(\mu)}} \delta, & y_{i}^{0} \approx s_{i}^{\frac{\rho_{i}^{1}(\mu)}{\eta_{i}(\mu)}} y_{i-1}^{1} \approx s_{i}^{\frac{\rho_{i}^{1}(\mu)}{\eta_{i}(\mu)}} \delta, \\
u_{i-1}^{1} \approx s_{i}^{\frac{B_{i}^{1}(\mu)}{\eta_{i}(\mu)}} u_{i}^{0} & v_{i}^{0} \approx s_{i}^{\frac{B_{i}^{2}(\mu)}{\eta_{i}(\mu)}} v_{i-1}^{1} .
\end{array}
$$

Let $F_{i}=F_{i}^{1} \circ F_{i}^{0}: S_{i-1}^{1} \rightarrow S_{i}^{1}$. Then $F_{i}$ is the Poincaré map induced by system (1.1) in some tubular neighborhood of the heteroclinic loop $\Gamma$.

By (3.2), (3.3), and (3.4), we get the expression of the map $F_{i}$ as follows:

$$
\left\{\begin{array}{l}
n_{i}^{1,1}=\left(w_{i}^{12}\right)^{-1} \delta s_{i}^{\frac{\rho_{i}^{1}(\mu)}{\eta_{i}(\mu)}}+M_{i}^{1} \mu+\text { h.o.t., } \\
n_{i}^{1,3}=u_{i}^{0}-\delta_{u_{i}}-w_{i}^{13}\left(w_{i}^{12}\right)^{-1} \delta s_{i}^{\frac{\rho_{i}^{1}(\mu)}{\eta_{i}(\mu)}}+M_{i}^{3} \mu+\text { h.o.t., } \\
n_{i}^{1,4}=\left(w_{i}^{44}\right)^{-1} s_{i}^{\frac{B_{i}^{2}(\mu)}{\eta_{i}(\mu)}} v_{i-1}^{1}+M_{i}^{4} \mu+\text { h.o.t.. }
\end{array}\right.
$$

Let $G_{i}\left(q_{i-1}^{1}\right)=\left(G_{i}^{1}, G_{i}^{3}, G_{i}^{4}\right)=F_{i}\left(q_{i-1}^{1}\right)-q_{i}^{1}, q_{0}^{1}=q_{2}^{1}$. Owing to (3.1) and (3.5), we have

$$
\left\{\begin{array}{l}
G_{i}^{1}=\delta\left[\left(w_{i}^{12}\right)^{-1}\left(s_{i}\right)^{\frac{\rho_{i}^{1}(\mu)}{\eta_{i}(\mu)}}-\left(s_{i+1}\right)^{\frac{\lambda_{i+1}^{1}(\mu)}{\eta_{i+1}(\mu)}}\right]+M_{i}^{1} \mu+\text { h.o.t., } \\
G_{i}^{3}=u_{i}^{0}-\delta_{u_{i}}-w_{i}^{13}\left(w_{i}^{12}\right)^{-1} \delta\left(s_{i}\right)^{\frac{\rho_{i}^{1}(\mu)}{\eta_{i}(\mu)}}-\left(w_{i}^{33}\right)^{-1}\left(s_{i+1}\right)^{\frac{B_{i+1}^{1}(\mu)}{\eta_{i+1}(\mu)}} u_{i+1}^{0}+M_{i}^{3} \mu+\text { h.o.t. } \\
G_{i}^{4}=-v_{i}^{1}+\delta_{v_{i}}+w_{i}^{14} \delta\left(s_{i+1}\right)^{\frac{\lambda_{i+1}^{1}(\mu)}{\eta_{i+1}(\mu)}}+\left(w_{i}^{44}\right)^{-1}\left(s_{i}\right)^{\frac{B_{i}^{2}(\mu)}{\eta_{i}(\mu)}} v_{i-1}^{1}+M_{i}^{4} \mu+\text { h.o.t.. }
\end{array}\right.
$$

Thus, there is a one to one correspondence between the two point heteroclinic loop, 1-homoclinic or 1-periodic orbit of (1.1), and the solution $Q=\left(s_{1}, u_{1}^{0}, v_{1}^{1}, s_{2}, u_{2}^{0}, v_{2}^{1}\right)$ of the following bifurcation equation with $s_{i} \geqslant 0, i=1,2$ :

$$
\left(\mathrm{G}_{1}^{1}, \mathrm{G}_{1}^{3}, \mathrm{G}_{1}^{4}, \mathrm{G}_{2}^{1}, \mathrm{G}_{2}^{3}, \mathrm{G}_{2}^{4}\right)=0 .
$$

\section{Bifurcation problems of 1-heteroclinic and 1-homoclinic orbits}

In this section, we study the existence and the uniqueness of 1-heteroclinic and 1-homoclinic orbit. Consider the solution of the bifurcation equation (3.7) at $Q=0$ and $\mu=0$, we have

$$
\begin{aligned}
G & \equiv \frac{\partial\left(G_{1}^{1}, G_{1}^{3}, G_{1}^{4}, G_{2}^{1}, G_{2}^{3}, G_{2}^{4}\right)}{\partial\left(s_{1}, s_{2}, u_{1}^{0}, u_{2}^{0}, v_{1}^{1}, v_{2}^{1}\right)} \\
& =\left(\begin{array}{cccccc}
\delta\left(w_{1}^{12}\right)^{-1} \frac{\rho_{1}^{1}(\mu)}{\eta_{1}(\mu)}\left(s_{1}\right)^{\frac{\rho_{1}^{1}(\mu)}{\eta_{1}(\mu)}-1} & -\delta \frac{\lambda_{2}^{1}(\mu)}{\eta_{2}(\mu)}\left(s_{2}\right)^{\frac{\lambda_{2}^{1}(\mu)}{\eta_{2}(\mu)}-1} & 0 & 0 & 0 & 0 \\
\delta \frac{w_{1}^{13}}{w_{1}^{12}} \frac{\rho_{1}^{1}(\mu)}{\eta_{1}(\mu)}\left(s_{1}\right)^{\frac{\rho_{1}^{1}(\mu)}{\eta_{1}(\mu)}-1} & 0 & 1 & 0 & 0 & 0 \\
0 & \delta w_{1}^{14} \frac{\lambda_{2}^{1}(\mu)}{\eta_{2}(\mu)}\left(s_{2}\right)^{\frac{\lambda_{2}^{1}(\mu)}{\eta_{2}(\mu)}-1} & 0 & 0 & -1 & 0 \\
-\delta \frac{\lambda_{1}^{1}(\mu)}{\eta_{1}(\mu)}\left(s_{1}\right)^{\frac{\lambda_{1}^{1}(\mu)}{\eta_{1}(\mu)}-1} & \delta\left(w_{2}^{12}\right)^{-1} \frac{\rho_{2}^{1}(\mu)}{\eta_{2}(\mu)}\left(s_{2}\right)^{\frac{\rho_{2}^{1}(\mu)}{\eta_{2}(\mu)}-1} & 0 & 0 & 0 & 0 \\
0 & \delta \frac{w_{2}^{13}}{w_{2}^{11}} \frac{\rho_{2}^{1}(\mu)}{\eta_{2}(\mu)}\left(s_{2}\right)^{\frac{\rho_{2}^{1}(\mu)}{\eta_{2}(\mu)}-1} & 0 & 1 & 0 & 0 \\
\delta w_{2}^{14} \frac{\lambda_{1}^{1}(\mu)}{\eta_{1}(\mu)}\left(s_{1}\right)^{\frac{\lambda_{1}^{1}(\mu)}{\eta_{1}(\mu)}-1} & 0 & 0 & 0 & 0 & -1
\end{array}\right) .
\end{aligned}
$$


Since $\eta_{i}(\mu)=\min \left\{\rho_{i}^{1}(\mu), \lambda_{i}^{1}(\mu)\right\}, \frac{\lambda_{i}^{1}(\mu)}{\eta_{i}(\mu)}-1$ and $\frac{\rho_{i}^{1}(\mu)}{\eta_{i}(\mu)}-1$ have only one zero, so, the rank of $G$ is at least 5 as $s_{1}=s_{2}=\mu=0$.

Moreover, if $\left(\rho_{1}^{1}-\lambda_{1}^{1}\right)\left(\rho_{2}^{1}-\lambda_{2}^{1}\right)>0$, then, by the continuity and $|\mu| \ll 1$, we have $\left(\rho_{1}^{1}(\mu)-\lambda_{1}^{1}(\mu)\right)\left(\rho_{2}^{1}(\mu)-\right.$ $\left.\lambda_{2}^{1}(\mu)\right)>0$ is always true, that is

$$
\begin{aligned}
& |\operatorname{det}(G)|=\left|\begin{array}{cccccc}
\delta\left(w_{1}^{12}\right)^{-1} \frac{\rho_{1}^{1}(\mu)}{\eta_{1}(\mu)}\left(s_{1}\right)^{\frac{\rho_{1}^{1}(\mu)}{\eta_{1}(\mu)}-1} & -\delta \frac{\lambda_{2}^{1}(\mu)}{\eta_{2}(\mu)}\left(s_{2}\right)^{\frac{\lambda_{2}^{1}(\mu)}{\eta_{2}(\mu)}-1} & 0 & 0 & 0 & 0 \\
-\delta \frac{\lambda_{1}^{1}(\mu)}{\eta_{1}(\mu)}\left(s_{1}\right)^{\frac{\lambda_{1}^{1}(\mu)}{\eta_{1}(\mu)}-1} & \delta\left(w_{2}^{12}\right)^{-1} \frac{\rho_{2}^{1}(\mu)}{\eta_{2}(\mu)}\left(s_{2}\right)^{\frac{\rho_{2}^{1}(\mu)}{\eta_{2}(\mu)}-1} & 0 & 0 & 0 & 0 \\
\delta \frac{w_{1}^{13}}{w_{1}^{12}} \frac{\rho_{1}^{1}(\mu)}{\eta_{1}(\mu)}\left(s_{1}\right)^{\frac{\rho_{1}^{1}(\mu)}{\eta_{1}(\mu)}-1} & 0 & 1 & 0 & 0 & 0 \\
0 & \delta \frac{w_{2}^{13}}{w_{2}^{12}} \frac{\rho_{2}^{1}(\mu)}{\eta_{2}(\mu)}\left(s_{2}\right)^{\frac{\rho_{2}^{1}(\mu)}{\eta_{2}(\mu)}-1} & 0 & 1 & 0 & 0 \\
0 & \delta w_{1}^{14} \frac{\lambda_{2}^{1}(\mu)}{\eta_{2}(\mu)}\left(s_{2}\right)^{\frac{\lambda_{2}^{1}(\mu)}{\eta_{2}(\mu)}-1} & 0 & 0 & -1 & 0 \\
\delta w_{2}^{14} \frac{\lambda_{1}^{1}(\mu)}{\eta_{1}(\mu)}\left(s_{1}\right)^{\frac{\lambda_{1}^{1}(\mu)}{\eta_{1}(\mu)}-1} & 0 & 0 & 0 & 0 & -1
\end{array}\right| \\
& =\delta^{2}\left(\left(w_{1}^{12} w_{2}^{12}\right)^{-1} \frac{\rho_{1}^{1}(\mu) \rho_{2}^{1}(\mu)}{\eta_{1}(\mu) \eta_{2}(\mu)}\left(s_{1}\right)^{\left(\frac{\rho_{1}^{1}(\mu)}{\eta_{1}(\mu)}-1\right)}\left(s_{2}\right)^{\left(\frac{\rho_{2}^{1}(\mu)}{\eta_{2}(\mu)}-1\right)}-\frac{\lambda_{1}^{1}(\mu) \lambda_{2}^{1}(\mu)}{\eta_{1}(\mu) \eta_{2}(\mu)}\left(s_{1}\right)^{\left(\frac{\lambda_{1}^{1}(\mu)}{\eta_{1}(\mu)}-1\right)}\left(s_{2}\right)^{\left(\frac{\lambda_{2}^{1}(\mu)}{\eta_{2}(\mu)}-1\right)}\right) \neq 0 .
\end{aligned}
$$

From the implicit function theorem, we have

Theorem 4.1. Suppose that (H1)-(H3) are valid, and for $|\mu|$ small enough, (3.7) has a unique solution about $s_{2}, \mu$

$$
s_{1}=s_{1}\left(s_{2}, \mu\right), u_{i}^{0}=u_{i}^{0}\left(s_{2}, \mu\right), v_{i}^{1}=v_{i}^{1}\left(s_{2}, \mu\right), i=1,2,
$$

or about $\mathrm{s}_{1}, \mu$

$$
s_{2}=s_{2}\left(s_{1}, \mu\right), u_{i}^{0}=u_{i}^{0}\left(s_{1}, \mu\right), v_{i}^{1}=v_{i}^{1}\left(s_{1}, \mu\right), i=1,2 .
$$

Specifically, if $\left(\rho_{1}^{1}-\lambda_{1}^{1}\right)\left(\rho_{2}^{1}-\lambda_{2}^{1}\right)>0$, then, (3.7) has a unique solution

$$
s_{i}=s_{i}(\mu), u_{i}^{0}=u_{i}^{0}(\mu), v_{i}^{1}=v_{i}^{1}(\mu), i=1,2,
$$

satisfying $s_{\mathfrak{i}}(0)=0, u_{\mathfrak{i}}^{0}(0)=0, v_{\mathfrak{i}}^{1}(0)=0, i=1,2$.

By Theorem 4.1, (3.6), and (3.7), it is easy to see that the equation $\left(\mathrm{G}_{1}^{3}, \mathrm{G}_{1}^{4}, \mathrm{G}_{2}^{3}, \mathrm{G}_{2}^{4}\right)=0$ always has a unique solution $u_{i}^{0}=u_{i}^{0}\left(s_{1}, s_{2}, \mu\right), v_{i}^{1}=v_{i}^{1}\left(s_{1}, s_{2}, \mu\right), i=1,2$, for $\delta,|\mu|$, and $s_{1}$, $s_{2}$ small enough. Substituting it into $\left(\mathrm{G}_{1}^{1}, \mathrm{G}_{2}^{1}\right)=0$, we get

$$
\left\{\begin{array}{l}
\left(s_{2}\right)^{\frac{\lambda_{2}^{1}(\mu)}{\eta_{2}(\mu)}}=\left(w_{1}^{12}\right)^{-1}\left(s_{1}\right)^{\frac{\rho_{1}^{1}(\mu)}{\eta_{1}(\mu)}}+\delta^{-1} M_{1}^{1} \mu+\text { h.o.t. } \\
\left(s_{1}\right)^{\frac{\lambda_{1}^{1}(\mu)}{\eta_{1}(\mu)}}=\left(w_{2}^{12}\right)^{-1}\left(s_{2}\right)^{\frac{\rho_{2}^{1}(\mu)}{\eta_{2}(\mu)}}+\delta^{-1} M_{2}^{1} \mu+\text { h.o.t.. }
\end{array}\right.
$$

Theorem 4.2. Suppose that (H1)-(H3) are valid, $\mathrm{M}_{i}^{1} \neq 0, i=1,2$, then the following are true:

(i) Near $\mu=0$, there exists a unique surface $L_{i}$ with codimension 1 and normal vector $M_{i}^{1}$ at $\mu=0$, such that system (1.1) has a heteroclinic orbit joining $p_{1}$ and $p_{2}$ near $\Gamma_{i}$ if and only if $\mu \in L_{i}$ and $|\mu| \ll 1$.

(ii) When $\operatorname{rank}\left(M_{1}^{1}, M_{2}^{1}\right)=2, L_{1}$ and $L_{2}$ are transversal at $\mu=0$. Let $\mathrm{L}_{12}=\mathrm{L}_{1} \cap \mathrm{L}_{2}$, which is codimension 2, the system (1.1) has a heteroclinic loop near $\Gamma$ for $\mu \in \mathrm{L}_{12}$ and $|\mu| \ll 1$, that is, $\Gamma$ is persistent.

Proof. If $s_{1}=s_{2}=0$, then (4.1) becomes

$$
\left\{\begin{array}{l}
M_{1}^{1} \mu+\text { h.o.t. }=0 \\
M_{2}^{1} \mu+\text { h.o.t. }=0
\end{array}\right.
$$

Thus, the necessary and sufficient condition for the persistence of $\Gamma_{i}$ is that (4.2) has solution.

If $M_{i}^{1} \neq 0$, then $M_{i}^{1} \mu+$ h.o.t. $=0, i=1,2$ has solution which defines a surface $L_{i}$ in the neighborhood of $\mu=0$. It is easy to see that $L_{i}$ has codimension 1 and a normal vector $M_{i}^{1}$ at $\mu=0$. 
$L_{1}$ and $L_{2}$ are transversal (resp. tangent) at $\mu=0$ if and only if $M_{1}^{1}$ and $M_{2}^{1}$ are linearly independent (resp. dependent). In the transversal case, i.e., $\operatorname{rank}\left(M_{1}^{1}, M_{2}^{1}\right)=2, L_{12}=L_{1} \cap L_{2}$ has a manifold structure near $\mu=0$ (see [2]). In fact, $L_{12}$ is a surface with codimension 2 such that (1.1) has heteroclinic loop near $\Gamma$ for $\mu \in \mathrm{L}_{12}$ and $|\mu| \ll 1$, that is, $\Gamma$ is persistent.

Denote $R_{1}=\left\{\mu: M_{1}^{1} \mu>0, \triangle_{2} M_{2}^{1} \mu<0,|\mu| \ll 1\right\}, R_{2}=\left\{\mu: M_{2}^{1} \mu>0, \triangle_{1} M_{1}^{1} \mu<0,|\mu| \ll 1\right\}$.

Theorem 4.3. Suppose that the hypotheses $(\mathrm{H} 1)-(\mathrm{H} 3)$ are valid. If the region $\mathrm{R}_{\mathrm{i}}$ is not empty, then, there exists a unique $(\mathrm{l}-1)$-dimensional surface $\widetilde{\mathrm{L}}_{i} \subset \mathrm{R}_{i}$, such that, for $\mu \in \widetilde{\mathrm{L}}_{i}$, system (1.1) has a 1-homoclinic orbit $\widetilde{\Gamma}_{i}$ connecting $p_{i}$, where, $i=1,2, \widetilde{\mathrm{L}}_{1}$, and $\widetilde{\mathrm{L}}_{2}$ are defined by

$$
\left(\delta^{-1} M_{1}^{1} \mu\right)^{\frac{\rho_{2}^{1}(\mu)}{\lambda_{2}^{1}(\mu)}}=\left(-\delta^{-1} w_{2}^{12} M_{2}^{1} \mu\right)+\text { h.o.t. }
$$

and

$$
\left(\delta^{-1} M_{2}^{1} \mu\right)^{\frac{\rho_{1}^{1}(\mu)}{\lambda_{1}^{1}(\mu)}}=\left(-\delta^{-1} w_{1}^{12} M_{1}^{1} \mu\right)+\text { h.o.t. }
$$

respectively. Moreover, $\widetilde{\mathrm{L}}_{i}$ has a normal vector $\mathrm{V}_{i}=\left\{\begin{array}{ll}\mathrm{M}_{i+1}^{1}, & \rho_{i+1}^{1}>\lambda_{i+1}^{1}, \\ M_{i}^{1}, & \rho_{i+1}^{1}<\lambda_{i+1}^{1},\end{array}\right.$ at $\mu=0$.

Proof. If $s_{\mathfrak{i}}=0, s_{i+1}>0$, then (4.1) becomes

$$
\left\{\begin{array}{l}
\left(s_{i+1}\right)^{\frac{\lambda_{i+1}^{1}(\mu)}{\eta_{i+1}(\mu)}}=\delta^{-1} M_{i}^{1} \mu+\text { h.o.t., } \\
\left(s_{i+1}\right)^{\frac{\rho_{i+1}^{1}(\mu)}{\eta_{i+1}(\mu)}}=-\delta^{-1} w_{i+1}^{12} M_{i+1}^{1} \mu+\text { h.o.t.. }
\end{array}\right.
$$

So, we have

$$
\left(\delta^{-1} M_{i}^{1} \mu\right)^{\frac{\rho_{i+1}^{1}(\mu)}{\lambda_{i+1}^{1}(\mu)}}=\left(-\delta^{-1} w_{i+1}^{12} M_{i+1}^{1} \mu\right)+\text { h.o.t., }
$$

which defines a surface $\widetilde{L}_{i}$ with codimension 1 in regions $R_{i}$.

It is easy to see that, if $\rho_{i+1}^{1}(\mu)>\lambda_{i+1}^{1}(\mu)\left(\rho_{i+1}^{1}(\mu)<\lambda_{i+1}^{1}(\mu)\right)$, then $\widetilde{L}_{i}$ has a normal vector $V_{i}=M_{i+1}^{1}$ $\left(M_{i}^{1}\right)$, which means $\widetilde{L}_{i}$ is tangent to $L_{i+1}\left(L_{i}\right)$ at $\mu=0$.

About the bifurcation diagrams, see Figures $3,4,5,6$.

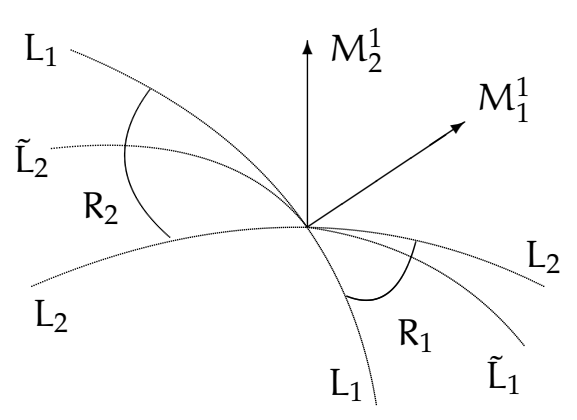

$$
\Delta_{1}=\Delta_{2}=1, \rho_{1}^{1}>\lambda_{1}^{1}, \rho_{2}^{1}>\lambda_{2}^{1}
$$

Figure 3

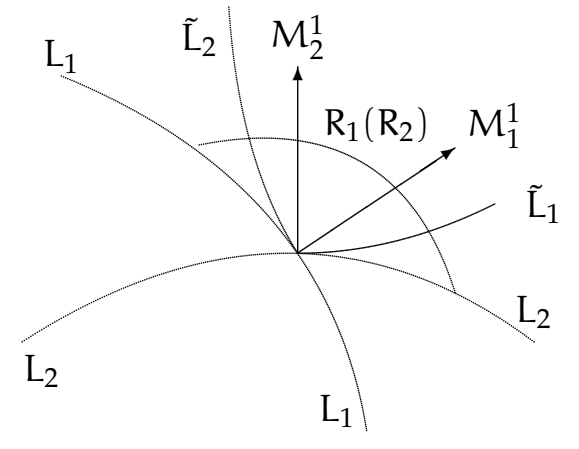

$\Delta_{1}=-1, \Delta_{2}=-1, \rho_{1}^{1}>\lambda_{1}^{1}, \rho_{2}^{1}>\lambda_{2}^{1}$

Figure 4 


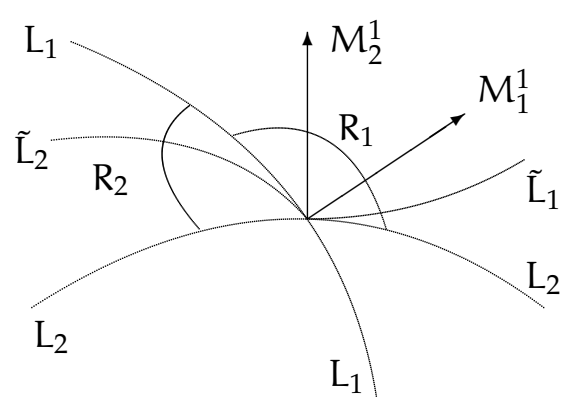

$\Delta_{1}=1, \Delta_{2}=-1, \rho_{1}^{1}>\lambda_{1}^{1}, \rho_{2}^{1}>\lambda_{2}^{1}$

Figure 5

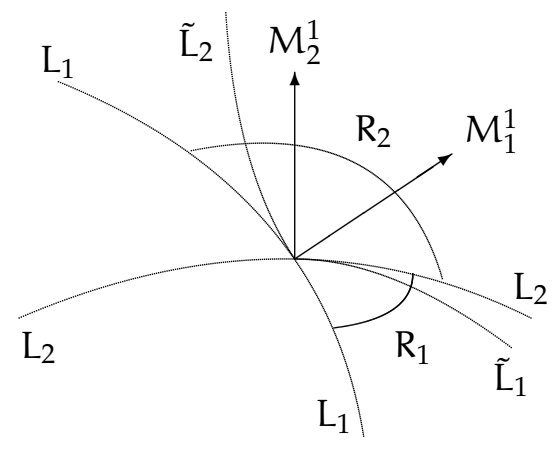

$\Delta_{1}=-1, \Delta_{2}=1, \rho_{1}^{1}>\lambda_{1}^{1}, \rho_{2}^{1}>\lambda_{2}^{1}$

Figure 6

\section{Bifurcation problems of 1-period orbits}

In this section, we discuss 1-period orbit bifurcation problems of $\Gamma$ as hyperbolic ratio $\beta_{i}=\rho_{i}^{1} / \lambda_{i}^{1}>1$, $i=1,2$, and locate the corresponding bifurcation surfaces. In other words, we study the solutions of (4.1) satisfying $s_{1}>0, s_{2}>0$.

(H4) Assume $\rho_{i}^{1}>\lambda_{i}^{1}, i=1,2$.

Denote $\beta_{\mathfrak{i}}(\mu)=\rho_{i}^{1}(\mu) / \lambda_{\mathfrak{i}}^{1}(\mu)>1, \beta_{\mathfrak{i}}=\beta_{\mathfrak{i}}(0), i=1,2$. In this case, the equation (4.1) turns to

$$
\left\{\begin{array}{l}
s_{2}=\left(w_{1}^{12}\right)^{-1} s_{1}^{\beta_{1}(\mu)}+\delta^{-1} M_{1}^{1} \mu+\text { h.o.t., } \\
s_{1}=\left(w_{2}^{12}\right)^{-1} s_{2}^{\beta_{2}(\mu)}+\delta^{-1} M_{2}^{1} \mu+\text { h.o.t.. }
\end{array}\right.
$$

From (5.1), we get

$$
\begin{aligned}
& s_{1}^{\beta_{1}(\mu)}+\delta^{-1} w_{1}^{12} M_{1}^{1} \mu+\text { h.o.t. }=w_{1}^{12}\left(w_{2}^{12}\left(s_{1}-\delta^{-1} M_{2}^{1} \mu+\text { h.o.t. }\right)\right)^{\frac{1}{\beta_{2}(\mu)}} \\
& s_{2}^{\beta_{2}(\mu)}+\delta^{-1} w_{2}^{12} M_{2}^{1} \mu+\text { h.o.t. }=w_{2}^{12}\left(w_{1}^{12}\left(s_{2}-\delta^{-1} M_{1}^{1} \mu+\text { h.o.t. }\right)\right)^{\frac{1}{\beta_{1}(\mu)}} .
\end{aligned}
$$

Let

$$
V_{1}\left(s_{1}\right)=s_{1}^{\beta_{1}(\mu)}+\delta^{-1} w_{1}^{12} M_{1}^{1} \mu+\text { h.o.t., } N_{1}\left(s_{1}\right)=w_{1}^{12}\left(w_{2}^{12}\left(s_{1}-\delta^{-1} M_{2}^{1} \mu+\text { h.o.t. }\right)\right)^{\frac{1}{\beta_{2}(\mu)}},
$$

and

$$
V_{2}\left(s_{2}\right)=s_{2}^{\beta_{2}(\mu)}+\delta^{-1} w_{2}^{12} M_{2}^{1} \mu+\text { h.o.t., } N_{2}\left(s_{2}\right)=w_{2}^{12}\left(w_{1}^{12}\left(s_{2}-\delta^{-1} M_{1}^{1} \mu+\text { h.o.t. }\right)\right)^{\frac{1}{\beta_{1}(\mu)}} .
$$

Case 1. (A1) $\Delta_{1}=\Delta_{2}=1$.

Obviously, if (A1) holds, we have

$$
R_{1}=\left\{\mu: M_{1}^{1} \mu>0, M_{2}^{1} \mu<0,|\mu| \ll 1\right\}, R_{2}=\left\{\mu: M_{2}^{1} \mu>0, M_{1}^{1} \mu<0,|\mu| \ll 1\right\} .
$$

Theorem 5.1. Suppose that the hypotheses (H1)-(H4) and (A1) are valid.

(i) The system (1.1) does not have any 1-period orbit, but has exactly one 1-homoclinic orbit $\widetilde{\Gamma}_{1}$ near $\Gamma$ as $\mu \in$ $\widetilde{\mathrm{L}}_{1} \subset \mathrm{R}_{1}$. And in $\mathrm{R}_{1}, \mathrm{~V}_{1}\left(\mathrm{~s}_{1}\right)$ is not tangent to $\mathrm{N}_{1}\left(\mathrm{~s}_{1}\right)$ at arbitrary $\mathrm{s}_{1}$ for $0<\mathrm{s}_{1},|\mu| \ll 1$.

(ii) The system (1.1) does not have any 1-period orbit, but has exactly one 1-homoclinic orbit $\widetilde{\Gamma}_{2}$ near $\Gamma$ as $\mu \in$ $\widetilde{\mathrm{L}}_{2} \subset \mathrm{R}_{2}$. And in $\mathrm{R}_{2}, \mathrm{~V}_{2}\left(\mathrm{~s}_{2}\right)$ is not tangent to $\mathrm{N}_{2}\left(\mathrm{~s}_{2}\right)$ at arbitrary $\mathrm{s}_{2}$ for $0<\mathrm{s}_{2},|\mu| \ll 1$. 
Proof. (i) By (5.2), we have

$$
\begin{aligned}
\dot{V}_{1}\left(s_{1}\right) & =\beta_{1}(\mu) s_{1}^{\beta_{1}(\mu)-1} \\
\dot{N}_{1}\left(s_{1}\right) & =\frac{1}{\beta_{2}(\mu)} w_{1}^{12}\left(w_{2}^{12}\right)^{\frac{1}{\beta_{2}(\mu)}}\left(s_{1}-\delta^{-1} M_{2}^{1} \mu+\text { h.o.t. }\right)^{\frac{1}{\beta_{2}(\mu)}-1} \\
& =\frac{1}{\beta_{2}(\mu)} w_{1}^{12} w_{2}^{12}\left(w_{2}^{12}\left(s_{1}-\delta^{-1} M_{2}^{1} \mu+\text { h.o.t. }\right)\right)^{\frac{1}{\beta_{2}(\mu)}-1} .
\end{aligned}
$$

By $\beta_{1}(\mu)>1>\frac{1}{\beta_{2}(\mu)}$, we have $\dot{N}_{1}\left(s_{1}\right)>\beta_{1}(\mu) s_{1}^{\frac{1}{\beta_{2}(\mu)}-1}>\dot{V}_{1}\left(s_{1}\right) \geqslant 0$ for $0 \leqslant s_{1} \ll 1$.

So, by Theorem 4.3 and the above inequality, we get $V_{1}(0)=N_{1}(0)$ and $\dot{N}_{1}\left(s_{1}\right)>\dot{V}_{1}\left(s_{1}\right)$ for $\mu \in \widetilde{L}_{1}$, $0 \leqslant s_{1} \ll 1$. Therefore, $V_{1}\left(s_{1}\right)<N_{1}\left(s_{1}\right)$ is always right for $s_{1}>0, \mu \in \widetilde{L}_{1}$. That is, the system (1.1) does not have any 1-period orbit for $\mu \in \widetilde{\mathrm{L}}_{1}$.

At the same time, $\dot{N}_{1}\left(s_{1}\right) \neq \dot{V}_{1}\left(s_{1}\right), 0 \leqslant s_{1} \ll 1$, which means $V_{1}\left(s_{1}\right)$ is not tangent to $N_{1}\left(s_{1}\right)$ at arbitrary $s_{1}$ for $0<s_{1},|\mu| \ll 1$ in $R_{1}$.

(ii) The proof is similar.

Due to the definitions of $L_{i}, R_{i}, \widetilde{L}_{i}$ and above lemma, we define some open regions. In $R_{1}$, open set $\left(R_{1}\right)_{0}$ is bounded by $L_{1}$ and $\widetilde{L}_{1}$, and open set $\left(R_{1}\right)_{1}$ is bounded by $L_{2}$ and $\widetilde{L}_{1}$. In $R_{2}$, open set $\left(R_{2}\right)_{0}$ is bounded by $L_{2}$ and $\widetilde{L}_{2}$, and open set $\left(R_{2}\right)_{1}$ is bounded by $L_{1}$ and $\widetilde{L}_{2}$.

Denote $D_{1}$ is the open region whose boundaries are $L_{1}$ and $L_{2}$, such that $D_{1} \bigcap\left\{\mu: M_{1}^{1} \mu>0, M_{2}^{1} \mu>\right.$ $0,|\mu| \ll 1\} \neq \emptyset$. $D_{0}$ is the open region whose boundaries are $L_{2}$ and $L_{1}$, such that $D_{0} \bigcap\left\{\mu: M_{1}^{1} \mu<0, M_{2}^{1} \mu<\right.$ $0,|\mu| \ll 1\} \neq \emptyset$.

We obtain the following theorem and the corresponding bifurcation figure.

Theorem 5.2. Suppose that hypotheses (H1)-(H4) and (A1) are valid, then the following conclusions are true.

(i) The system (1.1) has exactly one simple 1-period orbit near $\Gamma$ as $\mu \in\left(R_{1}\right)_{1}$.

(ii) The system (1.1) has exactly one 1-homoclinic loop homoclinic to $\mathrm{p}_{1}$ near $\Gamma$ as $\mu \in \widetilde{\mathrm{L}}_{1}$.

(iii) The system (1.1) does not have any 1-period orbit and 1-homoclinic loop near $\Gamma$ as $\mu \in\left(R_{1}\right)_{0}$.

(iv) The system (1.1) has exactly one simple 1-period orbit near $\Gamma$ as $\mu \in\left(R_{2}\right)_{1}$.

(v) The system (1.1) has exactly one 1-homoclinic loop homoclinic to $\mathrm{p}_{2}$ near $\Gamma$ as $\mu \in \widetilde{\mathrm{L}}_{2}$.

(vi) The system (1.1) does not have any 1-period orbit and 1-homoclinic loop near $\Gamma$ as $\mu \in\left(R_{2}\right)_{0}$.

(vii) The system (1.1) has exactly one simple 1-periodic orbits near $\Gamma$ as $\mu \in \mathrm{D}_{1}$.

(viii) The system (1.1) does not have any 1-periodic orbits near $\Gamma$ as $\mu \in \mathrm{D}_{0}$.

Proof. By (4.3) and (5.2), we know that for $\mu \in \tilde{L}_{1}, V_{1}\left(s_{1}\right)=N_{1}\left(s_{1}\right)$ has a unique solution $s_{1}=0$, that is, $\mathrm{V}_{1}(0)=\mathrm{N}_{1}(0)\left(\widetilde{\mathrm{L}}_{1}: \delta^{-1} \mathrm{M}_{1}^{1} \mu+\right.$ h.o.t. $\left.=\left(-\delta^{-1} w_{2}^{12} \mathrm{M}_{2}^{1} \mu+\text { h.o.t. }\right)^{\frac{1}{\beta_{2}(\mu)}}\right)$. Thus, the system (1.1) has exactly one 1-homoclinic loop homoclinic to $\mathrm{p}_{1}$ near $\Gamma$ as $\mu \in \widetilde{\mathrm{L}}_{1}$ (see Figure 7).

For $\mu \in\left(R_{1}\right)_{0}, V_{1}(0)=\delta^{-1} w_{1}^{12} M_{1}^{1} \mu+$ h.o.t. $<N_{1}(0)=w_{1}^{12}\left(-\delta^{-1} w_{2}^{12} M_{2}^{1} \mu+\text { h.o.t. }\right)^{\frac{1}{\beta_{2}(\mu)}}$, so, $V_{1}\left(s_{1}\right)=$ $\mathrm{N}_{1}\left(\mathrm{~s}_{1}\right)$ does not have any small solution satisfying $s_{1} \geqslant 0$. That is, the system (1.1) does not have any 1-period orbit and 1-homoclinic loop near $\Gamma$ as $\mu \in\left(R_{1}\right)_{0}$ (see Figure 8).

For $\mu \in\left(R_{1}\right)_{1}, V_{1}(0)=\delta^{-1} w_{1}^{12} M_{1}^{1} \mu+$ h.o.t. $>N_{1}(0)=w_{1}^{12}\left(-\delta^{-1} w_{2}^{12} M_{2}^{1} \mu+\text { h.o.t. }\right)^{\frac{1}{\beta_{2}(\mu)}}$, so, $V_{1}\left(s_{1}\right)=$ $N_{1}\left(s_{1}\right)$ has exactly one small solution satisfying $s_{1}>0$. That is, the system (1.1) has exactly one simple 1-period orbit near $\Gamma$ as $\mu \in\left(R_{1}\right)_{1}$ (see Figure 9). 
Similarly, By (4.4) and (5.3), we know that for $\mu \in \tilde{L}_{2}, V_{2}\left(s_{2}\right)=N_{2}\left(s_{2}\right)$ has a unique solution $s_{2}=0$, that is $\mathrm{V}_{2}(0)=\mathrm{N}_{2}(0)\left(\widetilde{\mathrm{L}}_{2}: \delta^{-1} M_{2}^{1} \mu+\right.$ h.o.t. $\left.=\left(-\delta^{-1} w_{1}^{12} M_{1}^{1} \mu+\text { h.o.t. }\right)^{\frac{1}{\beta_{1}(\mu)}}\right)$. Thus, the system (1.1) has exactly one 1-homoclinic loop homoclinic to $p_{2}$ near $\Gamma$ as $\mu \in \widetilde{\mathrm{L}}_{2}$.

For $\mu \in\left(R_{2}\right)_{0}, V_{2}(0)=\delta^{-1} w_{2}^{12} M_{2}^{1} \mu+$ h.o.t. $<N_{2}(0)=w_{2}^{12}\left(-\delta^{-1} w_{1}^{12} M_{1}^{1} \mu+\text { h.o.t. }\right)^{\frac{1}{\beta_{1}(\mu)}}$, so, $V_{2}\left(s_{2}\right)=$ $\mathrm{N}_{2}\left(s_{2}\right)$ does not have any small solution satisfying $s_{2} \geqslant 0$. That is, the system (1.1) does not have any 1-period orbit and 1-homoclinic loop near $\Gamma$ as $\mu \in\left(R_{2}\right)_{0}$.

For $\mu \in\left(R_{2}\right)_{1}, V_{2}(0)=\delta^{-1} w_{2}^{12} M_{2}^{1} \mu+$ h.o.t. $>N_{2}(0)=w_{2}^{12}\left(-\delta^{-1} w_{1}^{12} M_{1}^{1} \mu+\text { h.o.t. }\right)^{\frac{1}{\beta_{1}(\mu)}}$, so, $V_{2}\left(s_{2}\right)=$ $\mathrm{N}_{2}\left(s_{2}\right)$ has exactly one small solution satisfying $s_{2}>0$. That is, the system (1.1) has exactly one simple 1-period orbit near $\Gamma$ as $\mu \in\left(R_{2}\right)_{1}$.

For $\mu \in D_{0},(5.1)$ does not have any small solution satisfying $s_{1} \geqslant 0, s_{2} \geqslant 0$. That is, the system (1.1) does not have any 1-period orbit and 1-homoclinic loop near $\Gamma$ as $\mu \in \mathrm{D}_{0}$.

For $\mu \in D_{1}$, (5.1) has exactly one small solution satisfying $s_{1}>0, s_{2}>0$. That is, the system (1.1) has exactly one simple 1-period orbit near $\Gamma$ as $\mu \in D_{1}$.

About the bifurcation diagram, see Figure 10.

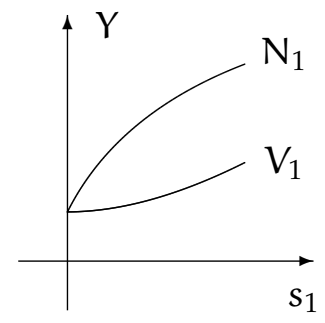

$\mu \in \tilde{\mathrm{L}}_{1}$

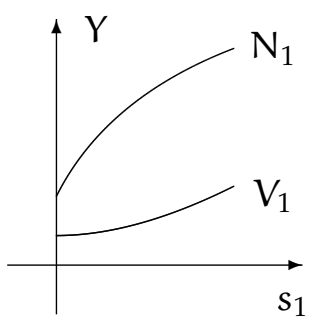

$\mu \in\left(R_{1}\right)_{0}$

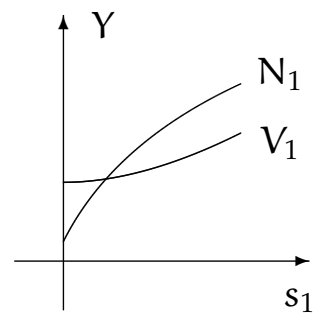

$\mu \in\left(R_{1}\right)_{1}$

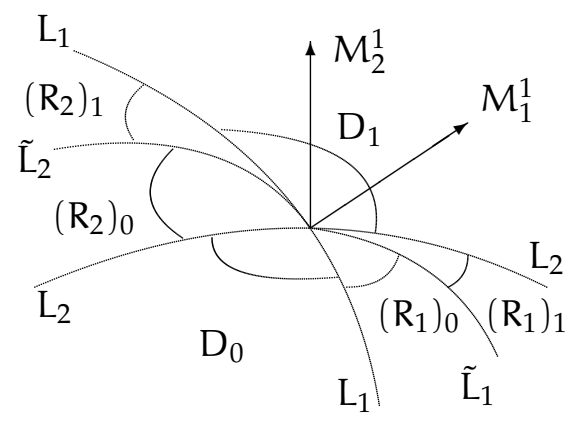

$\Delta_{1}=\Delta_{2}=1$

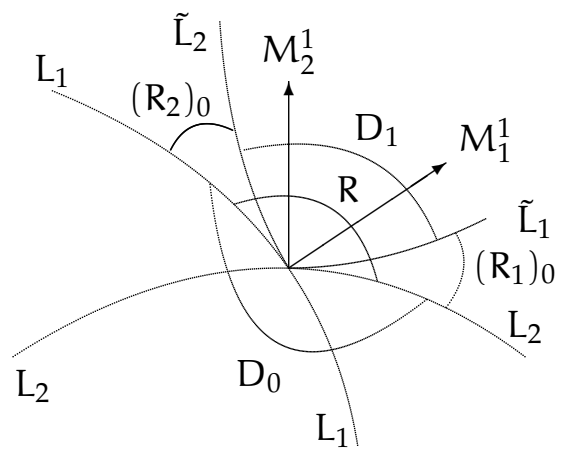

$\Delta_{1}=-1, \Delta_{2}=-1$

Figure 11

Case 2. (A2) $\Delta_{1}=-1, \Delta_{2}=-1$.

In this case, we have

$$
R=R_{1}=R_{2}=\left\{\mu: M_{1}^{1} \mu>0, M_{2}^{1} \mu>0,|\mu| \ll 1\right\} .
$$

Obviously, if $M_{1}^{1} \mu<0$ or $M_{2}^{1} \mu<0$, (5.1) does not have any non-negative solution except $s_{1}=s_{2}=0$. Similarly, we have 
Theorem 5.3. Suppose that the hypotheses (H1)-(H4) and (A2) are valid.

(i) The system (1.1) does not have any 1-period orbit, but has exactly one 1-homoclinic orbit $\widetilde{\Gamma}_{1}$ near $\Gamma$ as $\mu \in$ $\widetilde{\mathrm{L}}_{1} \subset \mathrm{R}$. In $\mathrm{R}, \mathrm{V}_{1}\left(\mathrm{~s}_{1}\right)$ is not tangent to $\mathrm{N}_{1}\left(\mathrm{~s}_{1}\right)$ at arbitrary $\mathrm{s}_{1}$ for $0<\mathrm{s}_{1},|\mu| \ll 1$.

(ii) The system (1.1) does not have any 1-period orbit, but has exactly one 1-homoclinic orbit $\widetilde{\Gamma}_{2}$ near $\Gamma$ as $\mu \in$ $\widetilde{\mathrm{L}}_{2} \subset \mathrm{R}$. In $\mathrm{R}, \mathrm{V}_{2}\left(\mathrm{~s}_{2}\right)$ is not tangent to $\mathrm{N}_{2}\left(\mathrm{~s}_{2}\right)$ at arbitrary $\mathrm{s}_{2}$ for $0<\mathrm{s}_{2},|\mu| \ll 1$.

Proof. (i) By (5.2), we get

$$
\dot{V}_{1}\left(s_{1}\right)=\beta_{1}(\mu) s_{1}^{\beta_{1}(\mu)-1}, \dot{N}_{1}\left(s_{1}\right)=\frac{1}{\beta_{2}(\mu)} w_{1}^{12} w_{2}^{12}\left(w_{2}^{12}\left(s_{1}-\delta^{-1} M_{2}^{1} \mu+\text { h.o.t. }\right)\right)^{\frac{1}{\beta_{2}(\mu)}-1} .
$$

By $\beta_{1}(\mu)>1>\frac{1}{\beta_{2}(\mu)}, \Delta_{1}=\Delta_{2}=-1$ and $M_{1}^{1} \mu>0, M_{2}^{1} \mu>0,|\mu| \ll 1$, it is easy to have

$$
\dot{N}_{1}\left(s_{1}\right)>\dot{V}_{1}\left(s_{1}\right) \geqslant 0 \text { for } 0 \leqslant s_{1} \ll 1 \text {. }
$$

So, by Theorem 4.3 and the above inequality, we get $V_{1}(0)=N_{1}(0)$ and $\dot{N}_{1}\left(s_{1}\right)>\dot{V}_{1}\left(s_{1}\right)$ for $\mu \in \widetilde{L}_{1}$, $0 \leqslant s_{1} \ll 1$. Therefore, $V_{1}\left(s_{1}\right)<N_{1}\left(s_{1}\right)$ is always right for $s_{1}>0, \mu \in \widetilde{L}_{1}$. That is, the system (1.1) does not have any 1-period orbit for $\mu \in \widetilde{\mathrm{L}}_{1}$.

At the same time, $\dot{N}_{1}\left(s_{1}\right) \neq \dot{V}_{1}\left(s_{1}\right), 0 \leqslant s_{1} \ll 1$, which means $V_{1}\left(s_{1}\right)$ is not tangent to $N_{1}\left(s_{1}\right)$ at arbitrary $s_{1}$ for $0<s_{1},|\mu| \ll 1$ in $R$.

(ii) The proof is similar.

In $R$, if (5.1) has solution $0<s_{1} \ll 1,0<s_{2} \ll 1$, then by (5.1), we know

$$
\begin{aligned}
-\delta^{-1} w_{2}^{12} M_{2}^{1} \mu>s_{2}^{\beta_{2}(\mu)} & =\left(\left(w_{1}^{12}\right)^{-1} s_{1}^{\beta_{1}(\mu)}+\delta^{-1} M_{1}^{1} \mu+\text { h.o.t. }\right)^{\beta_{2}(\mu)} \\
& >\left(\delta^{-1} M_{1}^{1} \mu+\text { h.o.t. }\right)^{\beta_{2}(\mu)}=\left.\left(-\delta^{-1} w_{2}^{12} M_{2}^{1} \mu\right)\right|_{\widetilde{L}_{1}},
\end{aligned}
$$

and

$$
\begin{aligned}
-\delta^{-1} w_{1}^{12} M_{1}^{1} \mu>s_{1}^{\beta_{1}(\mu)} & =\left(\left(w_{2}^{12}\right)^{-1} s_{2}^{\beta_{2}(\mu)}+\delta^{-1} M_{2}^{1} \mu+\text { h.o.t. }\right)^{\beta_{1}(\mu)} \\
& >\left(\delta^{-1} M_{2}^{1} \mu+\text { h.o.t. }\right)^{\beta_{1}(\mu)}=\left.\left(-\delta^{-1} w_{1}^{12} M_{1}^{1} \mu\right)\right|_{\tilde{L}_{2}} .
\end{aligned}
$$

Set $\left(R_{1}\right)_{0}$ is bounded by $L_{2}$ and $\widetilde{L}_{1}, D_{1}$ is bounded by $\widetilde{L}_{1}$ and $\widetilde{L}_{2}$, set $\left(R_{2}\right)_{0}$ is bounded by $\widetilde{L}_{2}$ and $L_{1}$, and they have nonempty intersection with $R$.

So, we have the following theorem and corresponding bifurcation diagram (see Figure 11).

Theorem 5.4. Suppose that the hypotheses (H1)-(H4) and (A2) are valid, then the following conclusions are true.

(i) The system (1.1) has exactly one simple 1-period orbit near $\Gamma$ as $\mu \in \mathrm{D}_{1}$.

(ii) The system (1.1) has exactly one 1-homoclinic loop homoclinic to $\mathrm{p}_{1}$ near $\Gamma$ as $\mu \in \widetilde{\mathrm{L}}_{1}$.

(iii) The system (1.1) does not have any 1-period orbit and 1-homoclinic loop near $\Gamma$ as $\mu \in\left(R_{1}\right)_{0}$.

(iv) The system (1.1) has exactly one 1-homoclinic loop homoclinic to $\mathrm{p}_{2}$ near $\Gamma$ as $\mu \in \widetilde{\mathrm{L}}_{2}$.

(v) The system (1.1) does not have any 1-period orbit and 1-homoclinic loop near $\Gamma$ as $\mu \in\left(R_{2}\right)_{0}$.

(vi) The system (1.1) does not have any 1-period orbit and 1-homoclinic loop near $\Gamma$ as $\mu \notin R$ which means that $\mu \in \mathrm{D}_{0}=\left\{\mu: M_{2}^{1} \mu<0,|\mu| \ll 1\right\} \cup\left\{\mu: M_{1}^{1} \mu<0,|\mu| \ll 1\right\}$. 
Case 3. (A3) $\Delta_{1}=1, \Delta_{2}=-1$.

If (A3) holds, we have

$$
R_{1}=\left\{\mu: M_{1}^{1} \mu>0, M_{2}^{1} \mu>0,|\mu| \ll 1\right\}, R_{2}=\left\{\mu: M_{2}^{1} \mu>0, M_{1}^{1} \mu<0,|\mu| \ll 1\right\} .
$$

Theorem 5.5. Suppose that the hypotheses (H1)-(H4) and (A3) are valid.

(i) The system (1.1) does not have any 1-period orbit, but has exactly one 1-homoclinic orbit $\widetilde{\Gamma}_{1}$ near $\Gamma$ as $\mu \in$ $\widetilde{\mathrm{L}}_{1} \subset \mathrm{R}_{1}$. And in $\mathrm{R}_{1}, \mathrm{~V}_{1}\left(\mathrm{~s}_{1}\right)$ is not tangent to $\mathrm{N}_{1}\left(\mathrm{~s}_{1}\right)$ at arbitrary $\mathrm{s}_{1}$ for $0<\mathrm{s}_{1},|\mu| \ll 1$.

(ii) The system (1.1) does not have any 1-period orbit, but has exactly one 1-homoclinic orbit $\widetilde{\Gamma}_{2}$ near $\Gamma$ as $\mu \in$ $\widetilde{\mathrm{L}}_{2} \subset \mathrm{R}_{2}$. And in $\mathrm{R}_{2}, \mathrm{~V}_{2}\left(\mathrm{~s}_{2}\right)$ is not tangent to $\mathrm{N}_{2}\left(\mathrm{~s}_{2}\right)$ at arbitrary $\mathrm{s}_{2}$ for $0<\mathrm{s}_{2},|\mu| \ll 1$.

Proof. (i) By (5.1), we have

$$
\begin{aligned}
& \left(\left(w_{1}^{12}\right)^{-1} s_{1}^{\beta_{1}(\mu)}+\delta^{-1} M_{1}^{1} \mu+\text { h.o.t. }\right)^{\beta_{2}(\mu)}=w_{2}^{12}\left(s_{1}-\delta^{-1} M_{2}^{1} \mu+\text { h.o.t. }\right), \\
& \left(\left(w_{2}^{12}\right)^{-1} s_{2}^{\beta_{2}(\mu)}+\delta^{-1} M_{2}^{1} \mu+\text { h.o.t. }\right)^{\beta_{1}(\mu)}=w_{1}^{12}\left(s_{2}-\delta^{-1} M_{1}^{1} \mu+\text { h.o.t. }\right) .
\end{aligned}
$$

Denote $V_{1}\left(s_{1}\right)$ and $N_{1}\left(s_{1}\right)$ are the left and right hand sides of (5.4), respectively, we have

$$
\dot{V}_{1}\left(s_{1}\right)=\beta_{2}(\mu) \beta_{1}(\mu)\left(w_{1}^{12}\right)^{-1} s_{1}^{\beta_{1}(\mu)-1}\left(\left(w_{1}^{12}\right)^{-1} s_{1}^{\beta_{1}(\mu)}+\delta^{-1} M_{1}^{1} \mu+\text { h.o.t. }\right)^{\beta_{2}(\mu)-1}, \dot{N}_{1}\left(s_{1}\right)=w_{2}^{12} .
$$

By $\beta_{1}(\mu)>1>\frac{1}{\beta_{2}(\mu)}, \Delta_{1}=1, \Delta_{2}=-1$ and $M_{1}^{1} \mu>0, M_{2}^{1} \mu>0,|\mu| \ll 1$, it is easy to have

$$
\dot{\mathrm{N}}_{1}\left(\mathrm{~s}_{1}\right)<0<\dot{\mathrm{V}}_{1}\left(\mathrm{~s}_{1}\right) \text {. }
$$

So, by Theorem 4.3 and the above inequality, we get $V_{1}(0)=N_{1}(0)$ and $\dot{N}_{1}\left(s_{1}\right)<\dot{V}_{1}\left(s_{1}\right)$ for $\mu \in \widetilde{L}_{1}$, $0 \leqslant s_{1} \ll 1$. Therefore, $\mathrm{N}_{1}\left(s_{1}\right)<\mathrm{V}_{1}\left(\mathrm{~s}_{1}\right)$ is always right for $s_{1}>0, \mu \in \widetilde{\mathrm{L}}_{1}$. That is, the system (1.1) does not have any 1-period orbit for $\mu \in \widetilde{\mathrm{L}}_{1}$.

At the same time, $\dot{N}_{1}\left(s_{1}\right) \neq \dot{V}_{1}\left(s_{1}\right), 0 \leqslant s_{1} \ll 1$, which means $V_{1}\left(s_{1}\right)$ is not tangent to $N_{1}\left(s_{1}\right)$ at arbitrary $s_{1}$ for $0<s_{1},|\mu| \ll 1$ in $R_{1}$.

(ii) Denote $V_{2}\left(s_{2}\right)$ and $N_{2}\left(s_{2}\right)$ are the left and right hand sides of (5.5), respectively, the proof is similar to that of (i).

In $R_{1}$, if (5.1) has solutions $0<s_{1} \ll 1,0<s_{2} \ll 1$, then by (5.1), we know

$$
\begin{aligned}
-\delta^{-1} w_{2}^{12} M_{2}^{1} \mu>s_{2}^{\beta_{2}(\mu)} & =\left(\left(w_{1}^{12}\right)^{-1} s_{1}^{\beta_{1}(\mu)}+\delta^{-1} M_{1}^{1} \mu+\text { h.o.t. }\right)^{\beta_{2}(\mu)} \\
& >\left(\delta^{-1} M_{1}^{1} \mu+\text { h.o.t. }\right)^{\beta_{2}(\mu)}=\left.\left(-\delta^{-1} w_{2}^{12} M_{2}^{1} \mu\right)\right|_{\widetilde{L}_{1}} .
\end{aligned}
$$

Set $\left(R_{1}\right)_{1}$ is bounded by $L_{1}$ and $\widetilde{L}_{1}$, set $\left(R_{1}\right)_{0}$ is bounded by $\widetilde{L}_{1}$ and $L_{2}$, and they have nonempty intersection with $R_{1}$.

In $R_{2}$, if (5.1) has solutions $0<s_{1} \ll 1,0<s_{2} \ll 1$, then by (5.1), we know

$$
\begin{aligned}
-\delta^{-1} w_{1}^{12} M_{1}^{1} \mu<s_{1}^{\beta_{1}(\mu)} & =\left(\left(w_{2}^{12}\right)^{-1} s_{2}^{\beta_{2}(\mu)}+\delta^{-1} M_{2}^{1} \mu+\text { h.o.t. }\right)^{\beta_{1}(\mu)} \\
& <\left(\delta^{-1} M_{2}^{1} \mu+\text { h.o.t. }\right)^{\beta_{1}(\mu)}=\left.\left(-\delta^{-1} w_{1}^{12} M_{1}^{1} \mu\right)\right|_{\widetilde{L}_{2}} .
\end{aligned}
$$

Set $\left(R_{2}\right)_{0}$ is bounded by $L_{2}$ and $\widetilde{L}_{2}$, set $\left(R_{2}\right)_{1}$ is bounded by $\widetilde{L}_{2}$ and $L_{1}$, and they have nonempty intersection with $R_{2}$.

So, we have the following theorem and corresponding bifurcation diagram (see Figure 12). 
Theorem 5.6. Suppose that the hypotheses (H1)-(H4) and (A3) are valid, then the following conclusions are true.

(i) The system (1.1) has exactly one simple 1-period orbit near $\Gamma$ as $\mu \in\left(R_{1}\right)_{1}$.

(ii) The system (1.1) has exactly one 1-homoclinic loop homoclinic to $\mathrm{p}_{1}$ near $\Gamma$ as $\mu \in \widetilde{\mathrm{L}}_{1}$.

(iii) The system (1.1) does not have any 1-period orbit and 1-homoclinic loop near $\Gamma$ as $\mu \in\left(R_{1}\right)_{0}$.

(iv) The system (1.1) has exactly one simple 1-period orbit near $\Gamma$ as $\mu \in\left(R_{2}\right)_{1}$.

(v) The system (1.1) has exactly one 1-homoclinic loop homoclinic to $\mathrm{p}_{2}$ near $\Gamma$ as $\mu \in \widetilde{\mathrm{L}}_{2}$.

(vi) The system (1.1) does not have any 1-period orbit and 1-homoclinic loop near $\Gamma$ as $\mu \in\left(R_{2}\right)_{0}$.

(vii) The system (1.1) does not have any 1-period orbit and 1-homoclinic loop near $\Gamma$ as $\mu \notin R_{1} \cup R_{2}$ which means $\mu \in \mathrm{D}_{0}=\left\{\mu: M_{2}^{1} \mu<0,|\mu| \ll 1\right\}$.

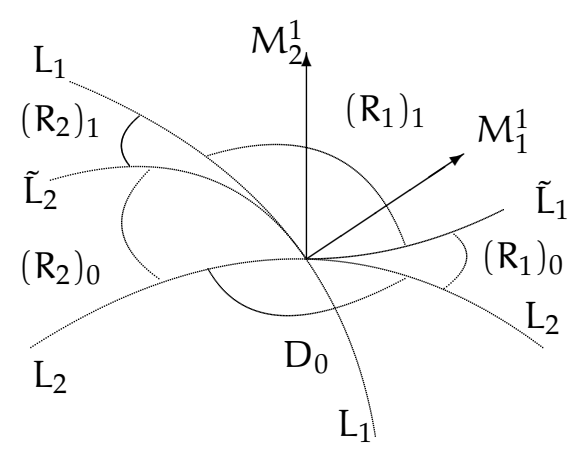

$$
\Delta_{1}=1, \Delta_{2}=-1
$$

Figure 12

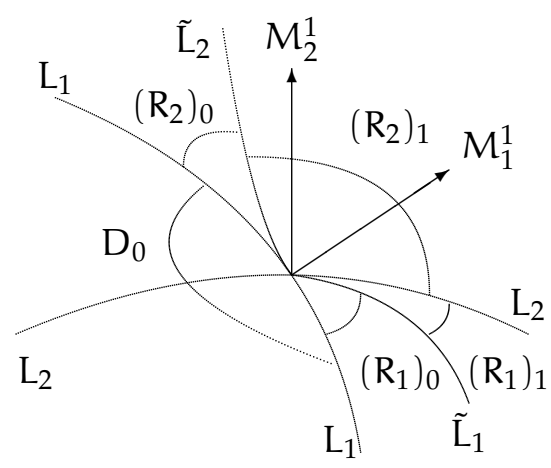

$\Delta_{1}=-1, \Delta_{2}=1$

Figure 13

Case 4. (A4) $\Delta_{1}=-1, \Delta_{2}=1$.

If (A4) holds, we have

$$
R_{1}=\left\{\mu: M_{1}^{1} \mu>0, M_{2}^{1} \mu<0,|\mu| \ll 1\right\}, \quad R_{2}=\left\{\mu: M_{2}^{1} \mu>0, M_{1}^{1} \mu>0,|\mu| \ll 1\right\} .
$$

Denote open set $\left(R_{1}\right)_{1}$ is bounded by $L_{2}$ and $\widetilde{L}_{1}$, set $\left(R_{1}\right)_{0}$ is bounded by $\widetilde{L}_{1}$ and $L_{1}$, and they have nonempty intersection with $R_{1}$; open set $\left(R_{2}\right)_{0}$ is bounded by $L_{1}$ and $\widetilde{L}_{2}$, set $\left(R_{2}\right)_{1}$ is bounded by $\widetilde{L}_{2}$ and $\mathrm{L}_{2}$, and they have nonempty intersection with $\mathrm{R}_{2}$.

Similarly, we have the following theorem and corresponding bifurcation diagram (see Figure 13)

Theorem 5.7. Suppose that hypotheses (H1)-(H4) and (A4) are valid, then the following conclusions are true.

(i) The system (1.1) has exactly one simple 1-period orbit near $\Gamma$ as $\mu \in\left(R_{1}\right)_{1}$.

(ii) The system (1.1) has exactly one 1-homoclinic loop homoclinic to $\mathrm{p}_{1}$ near $\Gamma$ as $\mu \in \widetilde{\mathrm{L}}_{1}$.

(iii) The system (1.1) does not have any 1-period orbit and 1-homoclinic loop near $\Gamma$ as $\mu \in\left(\mathrm{R}_{1}\right)_{0}$.

(iv) The system (1.1) has exactly one simple 1-period orbit near $\Gamma$ as $\mu \in\left(R_{2}\right)_{1}$.

(v) The system (1.1) has exactly one 1-homoclinic loop homoclinic to $\mathrm{p}_{2}$ near $\Gamma$ as $\mu \in \widetilde{\mathrm{L}}_{2}$.

(vi) The system (1.1) does not have any 1-period orbit and 1-homoclinic loop near $\Gamma$ as $\mu \in\left(R_{2}\right)_{0}$.

(vii) The system (1.1) does not have any 1-period orbit and 1-homoclinic loop near $\Gamma$ as $\mu \notin R_{1} \cup R_{2}$ which means $\mu \in \mathrm{D}_{0}=\left\{\mu: M_{1}^{1} \mu<0,|\mu| \ll 1\right\}$. 


\section{Bifurcation problems of 2-period orbits}

In this section, we discuss 2-period orbit bifurcation problems of $\Gamma$ as hyperbolic ratio $\beta_{i}=\rho_{i}^{1} / \lambda_{i}^{1}>1$, $i=1,2$ (Figure 14). Let $\tau_{3}, \tau_{4}$ be the flying times from $q_{2}^{3}\left(x_{2}^{3}, y_{2}^{3},\left(u_{2}^{3}\right)^{*},\left(v_{2}^{3}\right)^{*}\right) \in S_{2}^{1}$ to $q_{1}^{2}\left(x_{1}^{2}, y_{1}^{2},\left(u_{1}^{2}\right)^{*},\left(v_{1}^{2}\right)^{*}\right) \in$ $S_{1}^{0}, q_{1}^{3}\left(x_{1}^{3}, y_{1}^{3},\left(u_{1}^{3}\right)^{*},\left(v_{1}^{3}\right)^{*}\right) \in S_{1}^{1}$ to $q_{2}^{2}\left(x_{2}^{2}, y_{2}^{2},\left(u_{2}^{2}\right)^{*},\left(v_{2}^{2}\right)^{*}\right) \in S_{2}^{0}$, and $s_{3}=e^{-\eta_{1}(\mu) \tau_{3}}, s_{4}=e^{-\eta_{2}(\mu) \tau_{4}}$, respectively. We have have the bifurcation equation as following.

$$
\left\{\begin{array}{l}
\left(w_{1}^{12}\right)^{-1}\left(s_{1}\right)^{\beta_{1}(\mu)}-s_{2}+\delta^{-1} M_{1}^{1} \mu+\text { h.o.t. }=0 \\
\left(w_{2}^{12}\right)^{-1}\left(s_{2}\right)^{\beta_{2}(\mu)}-s_{3}+\delta^{-1} M_{2}^{1} \mu+\text { h.o.t. }=0 \\
\left(w_{1}^{12}\right)^{-1}\left(s_{3}\right)^{\beta_{1}(\mu)}-s_{4}+\delta^{-1} M_{1}^{1} \mu+\text { h.o.t. }=0 \\
\left(w_{2}^{12}\right)^{-1}\left(s_{4}\right)^{\beta_{2}(\mu)}-s_{1}+\delta^{-1} M_{2}^{1} \mu+\text { h.o.t. }=0 .
\end{array}\right.
$$

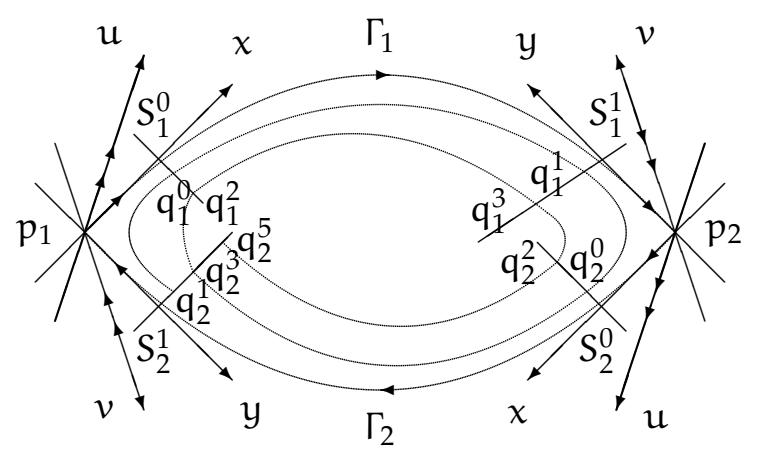

Figure 14

Due to $\rho_{1}^{1}>\lambda_{1}^{1}, \rho_{2}^{1}>\lambda_{2}^{1}$, we know that (6.1) has a unique solution $s_{1}=s_{1}(\mu), s_{2}=s_{2}(\mu), s_{3}=s_{3}(\mu)$, $s_{4}=s_{4}(\mu)$, satisfying $s_{1}(0)=0, s_{2}(0)=0, s_{3}(0)=0, s_{4}(0)=0$.

Theorem 6.1. Suppose that hypotheses (H1)-(H4) are valid, then, system (1.1) does not have any 2-heteroclinic loop which are heteroclinic to $\mathrm{p}_{1}$ and $\mathrm{p}_{2}$ near $\Gamma$ for $|\mu| \ll 1$.

Proof. If (6.1) has a solution $s_{1}>0, s_{2}>0, s_{3}=s_{4}=0$, then (6.1) becomes

$$
\left\{\begin{array}{l}
s_{2}=\left(w_{1}^{12}\right)^{-1}\left(s_{1}\right)^{\beta_{1}(\mu)}+\delta^{-1} M_{1}^{1} \mu+\text { h.o.t., } \\
\left(s_{2}\right)^{\beta_{2}(\mu)}+\delta^{-1} w_{2}^{12} M_{2}^{1} \mu+\text { h.o.t. }=0, \\
\delta^{-1} M_{1}^{1} \mu+\text { h.o.t. }=0 \\
s_{1}=\delta^{-1} M_{2}^{1} \mu+\text { h.o.t.. }
\end{array}\right.
$$

So,

$$
\begin{array}{r}
M_{2}^{1} \mu>0, \delta^{-1} M_{1}^{1} \mu+\text { h.o.t. }=0, w_{2}^{12}<0, w_{1}^{12}>0, \\
\left(w_{1}^{12}\right)^{-\beta_{2}(\mu)}\left(\delta^{-1} M_{2}^{1} \mu\right)^{\beta_{1}(\mu) \beta_{2}(\mu)}+\delta^{-1} w_{2}^{12} M_{2}^{1} \mu+\text { h.o.t. }=0 .
\end{array}
$$

By $\beta_{1}(\mu) \beta_{2}(\mu)>1$, we have

$$
M_{1}^{1} \mu+\text { h.o.t. }=0, M_{2}^{1} \mu+\text { h.o.t. }=0 .
$$

Thus, we have $s_{1}=0, s_{2}=0$, so, (1.1) does not have any 2-heteroclinic loop near $\Gamma$. 
Theorem 6.2. Suppose that hypotheses (H1)-(H4), (A3) (or (A4)) are valid, then, in $\left(\mathrm{R}_{1}\right)_{1}$, there exist an $(\mathrm{l}-1)$ dimensional surface $\tilde{\mathrm{L}}_{3}$ which is tangent to $\mathrm{L}_{2}$ at point $\mu=0$, such that system (1.1) has one 2-homoclinic loop homoclinic to $\mathrm{p}_{1}$ near $\Gamma$ for $\mu \in \tilde{\mathrm{L}}_{3},|\mu| \ll 1$ (see Figures 15 and 16). But in the cases (A1) and (A2), system (1.1) does not have any 2-homoclinic loop homoclinic to $p_{1}$ near $\Gamma$ for $|\mu| \ll 1$.

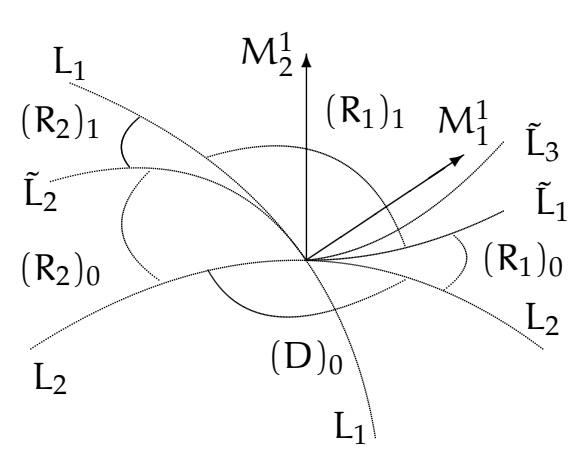

$$
\Delta_{1}=1, \Delta_{2}=-1
$$

Figure 15

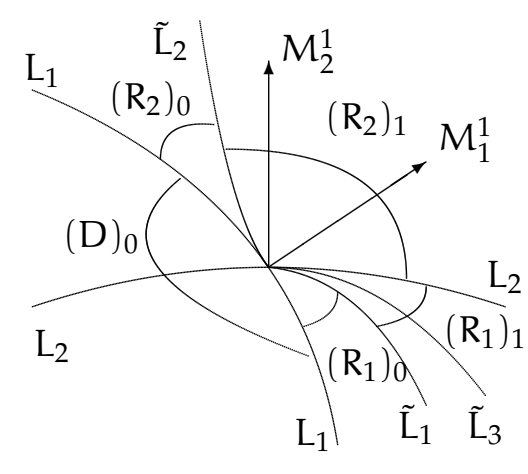

$$
\Delta_{1}=-1, \Delta_{2}=1
$$

Figure 16

Proof. If (6.1) has a solution $s_{1}>0, s_{2}>0, s_{3}=0, s_{4}>0$, then (6.1) becomes

$$
\left\{\begin{array}{l}
s_{1}=\left(w_{2}^{12}\right)^{-1}\left(s_{4}\right)^{\beta_{2}(\mu)}+\delta^{-1} M_{2}^{1} \mu+\text { h.o.t., } \\
s_{2}=\left(w_{1}^{12}\right)^{-1}\left(s_{1}\right)^{\beta_{1}(\mu)}+\delta^{-1} M_{1}^{1} \mu+\text { h.o.t., } \\
\left(s_{2}\right)^{\beta_{2}(\mu)}+\delta^{-1} w_{2}^{12} M_{2}^{1} \mu+\text { h.o.t. }=0, \\
s_{4}=\delta^{-1} M_{1}^{1} \mu+\text { h.o.t.. }
\end{array}\right.
$$

So, we have

$$
\begin{aligned}
\delta^{-1} M_{1}^{1} \mu & >0, \Delta_{2} M_{2}^{1} \mu<0 \\
s_{1} & =\left(w_{2}^{12}\right)^{-1}\left(\delta^{-1} M_{1}^{1} \mu\right)^{\beta_{2}(\mu)}+\delta^{-1} M_{2}^{1} \mu+\text { h.o.t. }>0 \\
s_{2} & =\left(w_{1}^{12}\right)^{-1}\left(s_{1}\right)^{\beta_{1}(\mu)}+\delta^{-1} M_{1}^{1} \mu+\text { h.o.t. } \\
& =\left(w_{1}^{12}\right)^{-1}\left[\left(w_{2}^{12}\right)^{-1}\left(\delta^{-1} M_{1}^{1} \mu\right)^{\beta_{2}(\mu)}+\delta^{-1} M_{2}^{1} \mu\right]^{\beta_{1}(\mu)}+\delta^{-1} M_{1}^{1} \mu+\text { h.o.t. }>0
\end{aligned}
$$

and

$$
\left\{\left(w_{1}^{12}\right)^{-1}\left[\left(w_{2}^{12}\right)^{-1}\left(\delta^{-1} M_{1}^{1} \mu\right)^{\beta_{2}(\mu)}+\delta^{-1} M_{2}^{1} \mu\right]^{\beta_{1}(\mu)}+\delta^{-1} M_{1}^{1} \mu\right\}^{\beta_{2}(\mu)}+\delta^{-1} w_{2}^{12} M_{2}^{1} \mu+\text { h.o.t. }=0 .
$$

Denote $\tilde{L}_{3}$ is the $(l-1)$-dimensional surface defined by (6.3), then, by (6.2) and (6.3), we know $\tilde{L}_{3}$ located in $\left(R_{1}\right)_{1}$ and tangent to $L_{2}$ at point $\mu=0$.

(i) If $\Delta_{1}=1, \Delta_{2}=-1$, then, by (6.3), we have

$$
-\delta^{-1} \mathcal{W}_{2}^{12} M_{2}^{1} \mu \|_{\tilde{L}_{3}}>\left(\delta^{-1} M_{1}^{1} \mu\right)^{\beta_{2}(\mu)}+\text { h.o.t. }=-\delta^{-1} w_{2}^{12} M_{2}^{1} \mu \|_{\tilde{L}_{1}},
$$

this means that $\tilde{L}_{3}$ is located in the region $\left(R_{1}\right)_{1}$. 
(ii) If $\Delta_{1}=-1, \Delta_{2}=1$, then, by (6.3), we have

$$
-\delta^{-1} \mathcal{W}_{2}^{12} M_{2}^{1} \mu \|_{\tilde{L}_{3}}<\left(\delta^{-1} M_{1}^{1} \mu\right)^{\beta_{2}(\mu)}+\text { h.o.t. }=-\delta^{-1} \mathcal{W}_{2}^{12} M_{2}^{1} \mu \|_{\tilde{L}_{1}}
$$

this means that $\tilde{L}_{3}$ is located in the region $\left(R_{1}\right)_{1}$.

(iii) For the case $\Delta_{1}=1, \Delta_{2}=1$, we know, if $\left(s_{1}, s_{2}\right)$ is a solution of (5.1), then, the duplication of it, $\left(s_{1}\right.$, $\left.s_{2}, s_{3}, s_{4}\right)=\left(s_{1}, s_{2}, s_{1}, s_{2}\right)$ must be the solutions of $(6.1)$ near $\left(s_{1}, s_{2}, s_{3}, s_{4}\right)=(0,0,0,0)$. Therefore, if $s_{3}=0, s_{4}>0$ satisfy (6.1), then (6.1) must have a solution $s_{1}=s_{3}=0, s_{2}=s_{4}>0$. By the uniqueness of the solution of (6.1), we get, system (1.1) has no 2-homoclinic loop homoclinic to $p_{1}$ near $\Gamma$ for $|\mu| \ll 1$.

(iv) For the case $\Delta_{1}=-1, \Delta_{2}=-1$, the reason is similar to that of $\Delta_{1}=1, \Delta_{2}=1$.

Thus, we get the theorem.

Theorem 6.3. Suppose that hypotheses (H1)-(H4), (A3) (or (A4)) are valid, then, in $\left(\mathrm{R}_{2}\right)_{1}$, there exist an $(\mathrm{l}-1)$ dimensional surface $\tilde{L}_{4}$ which is tangent to $\mathrm{L}_{1}$ at point $\mu=0$, such that system (1.1) has exactly one 2-homoclinic loop homoclinic to $\mathrm{p}_{2}$ near $\Gamma$ for $\mu \in \tilde{\mathrm{L}}_{4},|\mu| \ll 1$ (see Figures 17 and 18). But in the cases (A1) and (A2), system (1.1) has no 2-homoclinic loop homoclinic to $\mathrm{p}_{2}$ near $\Gamma$ for $|\mu| \ll 1$.

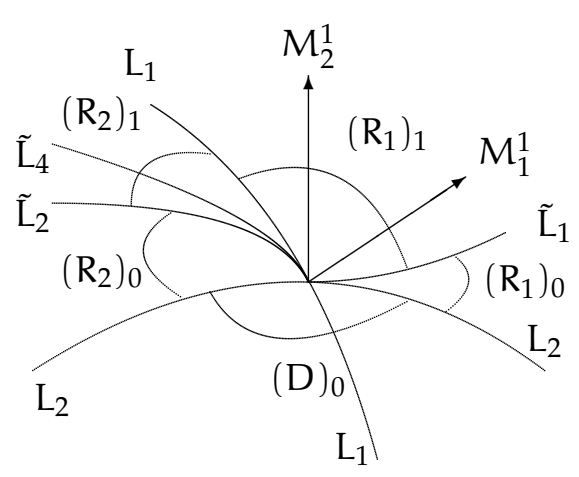

$$
\Delta_{1}=1, \Delta_{2}=-1
$$

Figure 17

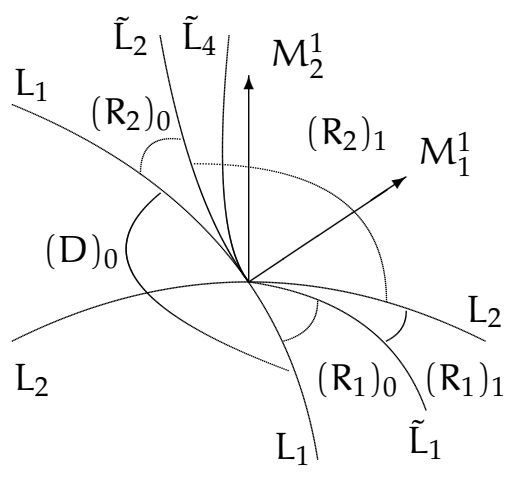

$\Delta_{1}=-1, \Delta_{2}=1$

Figure 18

Proof. If (6.1) has a solution $s_{1}>0, s_{2}>0, s_{3}>0, s_{4}=0$, then (6.1) becomes

$$
\left\{\begin{array}{l}
s_{1}=\delta^{-1} M_{2}^{1} \mu+\text { h.o.t., } \\
\left(s_{3}\right)^{\beta_{1}(\mu)}=-\delta^{-1} w_{1}^{12} M_{1}^{1} \mu+\text { h.o.t., } \\
s_{2}=\left(w_{1}^{12}\right)^{-1}\left(s_{1}\right)^{\beta_{1}(\mu)}+\delta^{-1} M_{1}^{1} \mu+\text { h.o.t., } \\
s_{3}=\left(w_{2}^{12}\right)^{-1}\left(s_{2}\right)^{\beta_{2}(\mu)}+\delta^{-1} M_{2}^{1} \mu+\text { h.o.t.. }
\end{array}\right.
$$

So

$$
\begin{aligned}
M_{2}^{1} \mu & >0, \Delta_{1} M_{1}^{1} \mu<0, \\
s_{2} & =\left(w_{1}^{12}\right)^{-1}\left(\delta^{-1} M_{2}^{1} \mu\right)^{\beta_{1}(\mu)}+\delta^{-1} M_{1}^{1} \mu+\text { h.o.t. }>0, \\
s_{3} & =\left(w_{2}^{12}\right)^{-1}\left(s_{2}\right)^{\beta_{2}(\mu)}+\delta^{-1} M_{2}^{1} \mu+\text { h.o.t. } \\
& =\left(w_{2}^{12}\right)^{-1}\left[\left(w_{1}^{12}\right)^{-1}\left(\delta^{-1} M_{2}^{1} \mu\right)^{\beta_{1}(\mu)}+\delta^{-1} M_{1}^{1} \mu\right]^{\beta_{2}(\mu)}+\delta^{-1} M_{2}^{1} \mu+\text { h.o.t. }>0,
\end{aligned}
$$


and

$$
\left\{\left(w_{2}^{12}\right)^{-1}\left[\left(w_{1}^{12}\right)^{-1}\left(\delta^{-1} M_{2}^{1} \mu\right)^{\beta_{1}(\mu)}+\delta^{-1} M_{1}^{1} \mu\right]^{\beta_{2}(\mu)}+\delta^{-1} M_{2}^{1} \mu\right\}^{\beta_{1}(\mu)}=-\delta^{-1} w_{1}^{12} M_{1}^{1} \mu+\text { h.o.t. }
$$

Denote $\tilde{L}_{4}$ is the $(l-1)$-dimensional surface defined by (6.5), then, by (6.4) and (6.5), we know $\tilde{L}_{4}$ is located in $\left(R_{2}\right)_{1}$ and tangent to $L_{1}$ at point $\mu=0$. Thus, we get the theorem.

Denote $D_{2}$ is a open region which is bounded by $\tilde{L}_{3}$ and $\tilde{L}_{4}, M_{1}^{1}$ points into $D_{2}$ from $\tilde{L}_{4}$, and $M_{2}^{1}$ also points into $\mathrm{D}_{2}$ from $\tilde{\mathrm{L}}_{3}$.

Theorem 6.4. Suppose that hypotheses (H1)-(H4), (A3) (or (A4)) are valid, then, for $\mu \in \mathrm{D}_{2}$ and $|\mu| \ll 1$, system (1.1) has exactly one 2-periodic loop near $\Gamma$. But in the cases (A1) and (A2), system (1.1) has no 2-periodic loop near $\Gamma$ for $|\mu| \ll 1$.

Proof. If (6.1) has a solution $s_{1}>0, s_{2}>0, s_{3}>0, s_{4}>0$, then (6.1) becomes

$$
\left\{\begin{array}{l}
s_{1}=\left(w_{2}^{12}\right)^{-1} s_{4}^{\beta_{2}(\mu)}+\delta^{-1} M_{2}^{1} \mu+\text { h.o.t., } \\
s_{2}=\left(w_{1}^{12}\right)^{-1} s_{1}^{\beta_{1}(\mu)}+\delta^{-1} M_{1}^{1} \mu+\text { h.o.t., } \\
s_{3}=\left(w_{2}^{12}\right)^{-1} s_{2}^{\beta_{2}(\mu)}+\delta^{-1} M_{2}^{1} \mu+\text { h.o.t., } \\
s_{4}=\left(w_{1}^{12}\right)^{-1} s_{3}^{\beta_{1}(\mu)}+\delta^{-1} M_{1}^{1} \mu+\text { h.o.t.. }
\end{array}\right.
$$

Differentiating both sides of the equations, we get

$$
\begin{aligned}
& \left(s_{3}\right)_{\mu}=\delta^{-1} M_{2}^{1}+O\left(\left(w_{2}^{12}\right)^{-1} s_{2}^{\beta_{2}(\mu)-1}\right)+\text { h.o.t., } \\
& \left(s_{4}\right)_{\mu}=\delta^{-1} M_{1}^{1}+O\left(\left(w_{1}^{12}\right)^{-1} s_{3}^{\beta_{1}(\mu)-1}\right)+\text { h.o.t.. }
\end{aligned}
$$

Thus, the directional derivatives of $s_{3}$ along $M_{2}^{1}$ at $\tilde{L}_{3}$ and $s_{4}$ along $M_{1}^{1}$ at $\tilde{L}_{4}$ are positive.

Notice that $s_{3}=0$ for $\mu \in \tilde{L}_{3}$, and $s_{4}=0$ for $\mu \in \tilde{L}_{4}$, by the monotonicity, we have $s_{3}>0, s_{4}>0$ for $\mu \in \mathrm{D}_{2}$.

So we get the results. 20).

Combining Theorems 6.1, 6.2, 6.3, and 6.4, we get the following bifurcation figures (Figures 19 and

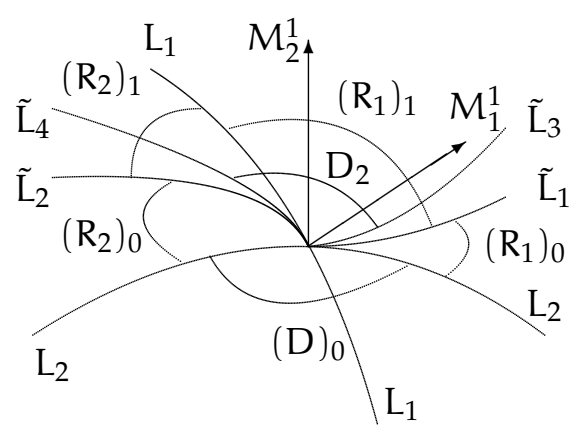

$$
\Delta_{1}=1, \Delta_{2}=-1
$$

Figure 19

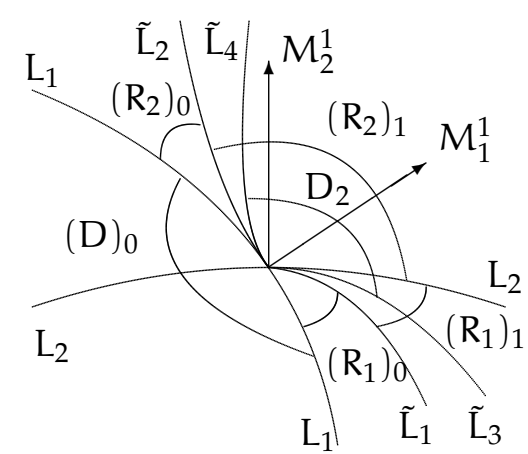

$$
\Delta_{1}=-1, \Delta_{2}=1
$$

Figure 20 


\section{Acknowledgment}

This work is supported by the National Natural Science Foundation of China (No. 11601212), Shandong Province Natural Science Foundation (ZR2015AL005), Shandong Province Higher Educational Science and Technology Program (J16LI03) and the Applied Mathematics Enhancement Program of Linyi University.

\section{References}

[1] R. Abraham, J. E. Marsden, T. Raţiu Manifolds, Tensor Analysis and Applications, Addison Wesley, London, (1983).1

[2] S. N. Chow, J. K. Hale, Methods of Bifurcation Theory, Springer-Verlag, New York, (1982). 4

[3] M. Han, D. Zhu, Bifurcation Theory of Differential Equations, Beijing, Coal Industry Press, (1994). 1

[4] Y. Jin, D. Zhu, Bifurcations of rough heteroclinic loops with two saddle points, Sci. China, Ser. A, 46 (2003), 459-468.1, $2,2,3$

[5] Y. Jin, X. Zhu, Z. Guo, H. Xu, L. Zhang, B. Ding, Bifurcations of Nontwisted Heteroclinic Loop with Resonant Eigenvalues, The Scientific World Journal, 2014 (2014), 8 pages. 1, 2, 3

[6] D. Luo, X. Wang, D. Zhu, M. Han, Bifurcation Theory and Methods of Dynamical Systems, World Scientific Publishing Co., Inc., River Edge, NJ, (1997). 1

[7] S. Wiggins, Global Bifurcations and Chaos-Analytical Methods, Springer, New York, (1988).

[8] S. Wiggins, Introduction to Applied Nonlinear Dynamical Systems and Chaos, Second Edition, Springer, New York, (2003).

[9] D. Zhu, Exponential trichotomy and heteroclinic bifurcation, Nonlinear Anal., 28 (1997), 547-557.1, 2, 3

[10] D. Zhu, Z. Xia, Bifurcations of heteroclinic loops, Sci. China Ser. A, 41 (1998), 837-848.1, 2, 3 